\title{
Molecular insights into antibody-mediated protection against the prototypic simian immunodeficiency virus
}

\begin{abstract}
AUTHORS: Fangzhu Zhao ${ }^{1,2,3,13}$, Zachary T. Berndsen²,3,5,13, Nuria Pedreño-Lopez ${ }^{4,13}$,

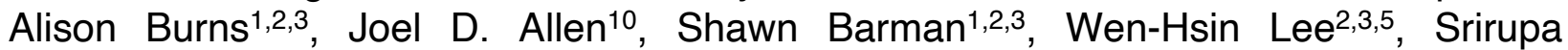
Chakraborty $^{9}$, Sandrasegaram Gnanakaran ${ }^{9}$, Leigh M. Sewall2,3,5, Gabriel Ozorowski2,3,5, Oliver Limbo 2,7, Ge Song1,2,3, Peter Yong ${ }^{1,2,3}$, Sean Callaghan ${ }^{1,2,3}$, Kim L. Weisgrau6, Jeffrey D. Lifson ${ }^{8}$, Rebecca Nedellec ${ }^{1,2,3}$, Thomas B Voigt ${ }^{4}$, Fernanda Laurino ${ }^{4}$, Johan Louw $^{4}$, Brandon C. Rosen ${ }^{4,11}$, Michael Ricciardi ${ }^{4}$, Max Crispin ${ }^{10}$, Ronald C. Desrosiers ${ }^{11}$,

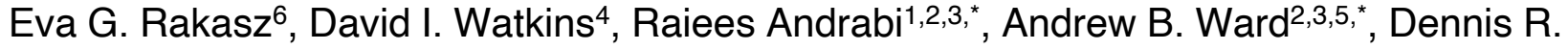
Burton $1,2,3,12,{ }^{*}$, Devin Sok $1,2,3,7,14,{ }^{*}$
\end{abstract}

\section{AFFILIATION}

1Department of Immunology and Microbiology, The Scripps Research Institute, La Jolla, CA 92037, USA

2IAVI Neutralizing Antibody Center, The Scripps Research Institute, La Jolla, CA 92037, USA

${ }^{3}$ Consortium for HIVIAIDS Vaccine Development (CHAVD), The Scripps Research Institute, La Jolla, CA 92037, USA

4Department of Pathology, George Washington University, Washington, DC 20037, USA ${ }^{5}$ Department of Integrative Structural and Computational Biology, The Scripps Research Institute, La Jolla, CA 92037, USA

${ }^{6}$ Wisconsin National Primate Research Center, University of Wisconsin-Madison, Madison, WI 53715, USA

${ }^{7}$ IAVI, New York, NY 10004, USA

${ }^{8} \mathrm{AIDS}$ and Cancer Virus Program, Frederick National Laboratory for Cancer Research, Frederick, MD 21701, USA

${ }^{9}$ Theoretical Biology and Biophysics Group, Los Alamos National Laboratory, Los Alamos, NM 87545, USA

${ }^{10}$ School of Biological Sciences, University of Southampton, Southampton, SO17 1BJ, UK

${ }^{11}$ Department of Pathology, Miller School of Medicine, University of Miami, Miami, FL 33136, USA

${ }^{12}$ Ragon Institute of Massachusetts General Hospital, Massachusetts Institute of Technology, and Harvard University, Cambridge, MA 02139, USA

${ }^{13}$ These authors contributed equally

${ }^{14}$ Lead Contact

*Correspondence: andrabi@scripps.edu (R.A.), andrew@scripps.edu (A.B.W.),

burton@scripps.edu (D.R.B.), dsok@iavi.org (D.S.) 


\section{SUMMARY:}

SIVmac239 infection of macaques is a favored model of human HIV infection. However, the SIVmac239 envelope (Env) trimer structure, glycan occupancy, and the targets and ability of neutralizing antibodies (nAbs) to protect against SIVmac239 remain unknown. Here, we report the isolation of SIVmac239 nAbs that recognize a glycan hole and the V1/V4 loop. A high-resolution structure of a SIVmac239 Env trimer-nAb complex shows many similarities to HIV and SIVcpz Envs, but with distinct V4 features and an extended V1 loop. Moreover, SIVmac239 Env has a higher glycan shield density than HIV Env that may contribute to poor or delayed $\mathrm{nAb}$ responses in SIVmac239-infected macaques. Passive transfer of a $\mathrm{nAb}$ protects macaques from repeated low dose intraveneous SIVmac239 challenge at serum titers comparable to those described for protection of humans against HIV infection. Our results provide structural insights for vaccine design and shed light on antibody-mediated protection in the SIV model. 


\section{INTRODUCTION:}

Since its first description in 1985, Simian Immunodeficiency Virus (SIV) infection of monkeys has been routinely used as a model for HIV infection of humans (Daniel et al., 1985; Barouch et al., 2012; Reynolds et al., 2008; Roederer et al., 2014). The molecular clone, SIVmac239, that infects rhesus macaques has been widely used in vaccination and protection studies (Casimiro et al., 2005; Daniel et al., 1992; Fuchs et al., 2015; Martins et al., 2019; Poignard et al., 2012; Wilson et al., 2006). The efficacy of several vaccine constructs and vaccine regimens against mucosal challenge by SIVmac239 in macaques has preceded their investigation in HIV vaccine clinical trials. Many of these SIV vaccines have focused on inducing $T$ cell responses alone or $T$ cell responses in combination with non-neutralizing antibody responses. The most successful of the macaque vaccines has been the rhesus cytomegalovirus (RhCMV)-SIV protocol that induces $\mathrm{MHC}$ class E-restricted $\mathrm{CD}^{+}{ }^{+} \mathrm{T}$ cells and results in early complete arrest of SIVmac239 replication and subsequent viral clearance in 50-60\% of animals (Hansen et al., 2013, 2019).

In contrast to $T$ cell responses, limited studies have investigated $n A b$ responses to SIVmac239 and their potential protective activities. This paucity likely reflects the observation that $n A b$ responses in natural SIVmac239 infection are relatively weak or delayed such that isolating monoclonal nAbs is difficult (Montefiori et al., 1996; PedreñoLopez et al., 2020). Furthermore, there are no immunogens that have been described capable of inducing even autologous nAbs to SIVmac239. Conversely, monoclonal nAbs isolated from HIV infection and SHIV-infected macaques have been critical in establishing the conditions for protection by nAbs against HIV in humans and small animal models and against chimeric simian human immunodeficiency virus (SHIV) in macaques (Julg et al., 2017; Pegu et al., 2014, 2019; Shingai et al., 2014).

There is strong and increasing interest in combining $T$ cell and nAb responses in an HIV vaccine (Chanzu and Ondondo, 2014; McMichael and Koff, 2014). This interest arises from the partial success of the RhCMV-SIV strategy described above and the results from the HVTN703/HPTN081 and HVTN704/HPTN085 Antibody Mediated Protection (AMP) study that show relatively high $\mathrm{nAb}$ titers are required for passive antibody protection against HIV challenge (Corey et al., 2021). Synergy between the two arms of the immune system could lead to more effective protection than achieved with one arm only. Indeed, 
synergy has been reported for protection against SHIV challenge in macaques; a reduction in the serum $n A b$ titer associated with protection was noted in the presence of a $\mathrm{T}$ cell response induced by immunization with a heterologous viral vector regime (Arunachalam et al., 2020). As above however, although improvements have been made to the SHIV model (Asmal et al., 2015; Del Prete et al., 2014; Harouse et al., 1999), the SIV model is the one that has been most explored and is believed the best mimic of the diversity that arises through HIV infection in humans (Watkins et al., 2008). Thus, to fully explore of $\mathrm{T}$ cell and $\mathrm{nAb}$ synergy in the SIVmac239 model requires $\mathrm{nAbs}$ and/or immunogens able to induce nAbs to the virus.

Here, we report the isolation of 12 potent SIVmac239 monoclonal nAbs from three chronically infected rhesus macaques and map their binding specificities on the SIVmac239 Env trimer. We show that passive transfer of the nAb K11 protects against SIVmac239 challenge in rhesus macaques using a repeat intravenous challenge route with $1 \mathrm{AID}_{50}$ virus dose. We also present the high-resolution structure of a recombinant SIVmac239 trimer in complex with nAb K11 by electron cryo-microscopy (cryo-EM) and find that it has many features in common with the HIV-1 and SIVcpz Env trimer structures solved to date, along with some distinctive features. To complement the cryo-EM structure, we also determined the identity of all $\mathrm{N}$-linked glycans via mass spectrometry (MS) and use this combined data to carry out computational modeling of fully glycosylated trimers. Our results corroborate the role of the glycan shield in limiting the elicitation of nAbs, highlight the importance of nAbs in protection against SIV infection, provide a structure for the nAb SIV vaccine target and pave the way for studying the combination of $T$ cells and antibodies in the SIVmac239 challenge model.

\section{RESULTS}

\section{Isolation of neutralizing antibodies from SIVmac239-infected rhesus macaques}

We screened infected rhesus macaques for plasma neutralization activity against the SIVmac239 pseudovirus (Figure S1A)(Pedreño-Lopez et al., 2020) and selected three ( $r 11039, r 11008$, and $r 11002$ ) with the most potent activity that have been infected for 6 to 10 months for $n A b$ generation. First, IgG $^{+}$memory $B$ cells from peripheral blood mononuclear cells (PBMCs) were antigen-selected using a recombinant SIVmac239 
SOSIP.664 trimer (von Bredow et al., 2019) (Figure 1A and Figure S1B). An average of $1.62 \%$ of $\mathrm{CD}^{2} 0^{+} \operatorname{lgM} \mathrm{M}^{-} \operatorname{lgG}{ }^{+}$memory $\mathrm{B}$ cells bound to the SIVmac239 trimer antigen. Antigen-specific $B$ cells were single-cell sorted and cultured in 384 well plates (Huang et al., 2013; Zhao et al., 2020). A total of 8470 single cells were sorted and expanded in vitro, from which 1546 wells secreted detectable IgG in supernatants. Around $52 \%$ of the $\operatorname{lgG}+$ wells showed measurable binding to SIVmac239 SOSIP protein by enzyme-linked immunosorbent assay (ELISA) screening (data not shown) but only 20 wells were positive for SIVmac239 pseudovirus micro-neutralization. The frequency of autologous nAb hits identified by supernatant screening for SIVmac239 was lower than SHIVBG505 infected macaques $(0.26 \%$ and $1.18 \%$ using $80 \%$ neutralization cut off, respectively) (data adapted from (Zhao et al., 2020)). Hence, the majority of SIV Env-specific memory B cells target non-neutralizing epitopes, likely more so than SHIV or HIV (Tomaras et al., 2008).

We cloned and expressed 12 monoclonal antibodies (mAbs) and annotated to the rhesus macaque germline database (Cirelli et al., 2019), where we identified 3 clonally related lineages, numbered 1 to 3 (Figure 1B). The SIV mAbs have an average of $10.8 \%$ somatic hypermutation rate (SHM) in the heavy chain and $6.4 \%$ in the light chain at the nucleotide level (Figure S1C). The third complementarity determining region of the heavy chain (CDRH3) in lineages 1 and 2 is relatively long, at 25 and 18 amino acids, respectively, whereas lineage 3 has a closer to average CDRH3 length of 13 amino acids (Figure 1B) (Francica et al., 2015; Sundling et al., 2012).

All 12 mAbs neutralized the tier 3 SIVmac239 pseudovirus in the TZM-bl assay (Figure 1C) with the most potent antibody FZ019.2 neutralizing at an $\mathrm{IC}_{50}$ value of $0.01 \mu \mathrm{g} / \mathrm{mL}$ (Figure 1D). However, lineage 3 antibodies FZ019.2 and J9 showed incomplete neutralization ( $90 \%$ and $72 \%$ respectively) at the highest antibody concentration (50 $\mu \mathrm{g} / \mathrm{mL}$ ) (Figures 1C and 1D). In contrast, antibodies in lineages 1 and 2 fully neutralized SIVmac239 at a median $\mathrm{IC}_{50}$ value of $0.80 \mu \mathrm{g} / \mathrm{mL}$ (Figures $1 \mathrm{C}$ and $1 \mathrm{D}$ ). The lineage 1 $\mathrm{K} 11$ antibody neutralized SIVmac239 at an $\mathrm{IC}_{50}$ of $0.24 \mu \mathrm{g} / \mathrm{mL}$, which is 5-fold more potent than ITS90.03, the only reported SIVmac239 nAb lineage so far (Gorman et al., 2019) (Figure 1D).

\section{Rhesus nAbs target a glycan hole epitope and the V4 loop on the SIVmac239 Env}


To map the binding specificity of isolated mAbs, we performed ELISA and included the non-neutralizing antibody (non-nAb) $5 \mathrm{~L} 7$ for comparison (Fuchs et al., 2015). All mAbs from lineages 1 and 2 bound to SIVmac239 SOSIP.664, SIVmac239 gp120, SIVmac239 gp140 foldon trimer (FT) (Gorman et al., 2019), and gp140 FT dV1V2V3 core proteins, whereas FZ019.2 and J9 showed decreased binding activity against gp120 dV1V2 proteins (Table S1).

Competition ELISA revealed that antibodies in lineages 1 and 2 target a similar epitope as ITS90.03 (Figure 2A). ITS90.03 has previously been shown to recognize a glycan hole on SIVmac239 gp120 Env centered on K254 (numbered by SIVmac239, 238 numbered by HIV HxB2) (Gorman et al., 2019). Thus, it appears that the glycan hole is a notable neutralizing epitope among SIVmac239-infected rhesus macaques. The lineage 3 nAbs, FZ019.2 and J9, did not compete with ITS90.03 nor did they compete with CD4-IgG2. Of note, the SIVmac239 SOSIP trimer that we used contains a K180S substitution in the V2 loop to increase binding affinity of the V2 apex-specific HIV-1 broadly neutralizing antibody (bnAb) PGT145, which enables its use in competition experiments (von Bredow et al., 2019; Walker et al., 2011). The lineage 3 nAbs also did not compete with PGT145 for Env trimer binding suggesting a distinct epitope that is not V2 apex or CD4 binding site (CD4bs) (Figure S2A). Evaluation of lineage 3 antibodies against glycosidase-treated SIVmac239 pseudovirus in neutralization assays indicate a dependence of these antibodies on hybrid-type or complex-type glycans to some degree (Figure S2B).

We used single-particle negative-stain electron microscopy (nsEM) to further characterize antibody epitope specificities by complexing with SIVmac239 SOSIP.664 Env trimer. The $3 \mathrm{D}$ reconstructions revealed three $\mathrm{K} 11$ antibody molecules bound to the trimer (Figure $2 \mathrm{~A})$. Consistent with the competition ELISA data, K11 recognized a similar glycan hole epitope as ITS90.03 (Gorman et al., 2019). ITS90.03 failed to neutralize the SIVmac239 K254N mutant, which restores the $\mathrm{N}$-link glycan sequon (Asn-X-Ser/Thr, where $\mathrm{X}$ is any amino acid except proline) at that position. Similarly, K11 showed dramatically decreased neutralization activity against this virus mutant (Figure $2 \mathrm{~B}$ ). By contrast, neutralization of FZ019.2 was not affected by mutations at the 254 position (Figure 2B), and nsEM revealed that FZ019.2 binds near the V4 loop on SIVmac239 Env (Figures 2C, 2D, S3A, and S3B). Alanine-scanning mutagenesis revealed that several V4 residues including 
N415, T416, N418, Q419, and P421 are important for FZ019.2 neutralization (Figure $\mathrm{S} 2 \mathrm{C})$.

We further tested neutralization breadth of mAbs against a panel of SIV pseudoviruses (Gorman et al., 2019; Kilgore et al., 2015). As expected, the neutralization breadth of glycan hole-targeting antibodies depended on the residue at position 254 on gp120: most viruses harboring the glycan hole were sensitive to neutralization while other viruses with an N-linked glycan sequon at position 254 were more neutralization resistant (Figure 2D). In contrast, FZ019.2 and J9 nAbs only neutralized autologous SIVmac239 and SIVmac239.cs.23 isolates (Figure 2D), which is as expected since the V4 loop is poorly conserved between isolates.

\section{Non-neutralizing SIVmac239 epitopes are immunodominant}

To assess epitope specificities for non-nAbs to SIVmac239, we isolated $7 \mathrm{mAbs}$ that bound SIVmac239 Env proteins but did not neutralize the autologous virus (Figures S2D and S2E). We similarly performed ELISA epitope mapping and competition assays. These data reveal that the majority of the non-nAbs recognize a similar specificity to $5 \mathrm{~L} 7$ (Figure S2D). These antibodies target an epitope that is not dependent on the V1, V2, and V3 loops on gp120 (Figure S2F). nsEM indicates that the prototype FZ012.7 antibody binds near the V4 and V5 loops (Figure S3E). Antibodies FZ012.14 and FZ012.16 appear to recognize the V3 loop on SIVmac239 Env as they bind SIVmac239 gp120 but fail to bind SIVmac239 delta V3 protein (Figure S2F).

To further elaborate the antigenic surface of SIVmac293 Env, we mapped the epitopes targeted in polyclonal serum responses among 9 infected rhesus macaques. As shown in Figures S1A, 4 macaques developed nAb responses against SIVmac239 (r11008, r11002, r11039, r11004) and 5 displayed undetectable serum nAb titers (Rh33519, Rh31186, Rh34620, Rh32388, Rh34118). All animals showed serum binding titers to SIVmac239 SOSIP. We performed serum competition assays with SIVmac239 SOSIP as antigen and observed that all infected sera strongly competed for binding with antibodies that recognize the 5L7/FZ012.7 epitope and a few animals competed for binding to the V3 loop (Figure 3A). None of the sera showed strong competition with PGT145, CD4IgG2, FZ019.2, or K11 for binding to SIVmac239 SOSIP, suggesting that the neutralizing 
epitopes are relatively poorly targeted in natural infection and consistent with relatively low serum neutralizing titers.

Additionally, all the infected sera bound overlapping SIVmac239 Env 15-mer peptides on V1V2, V3 and gp41 (Figure 3B), whereas non-infected serum and SIVmac239 nAbs did not. We further analyzed the polyclonal antibody binding specificities in infected sera with electron microscopy polyclonal epitope mapping (EMPEM) (Bianchi et al., 2018). 2-D classification revealed a high proportion of non-trimeric Env fragments that were extensively decorated with polyclonal Fabs (Figures $3 \mathrm{C}$ and S3F). These degraded trimers could result from inherent instability of SIVmac239 SOSIP and/or antibodyinduced trimer disassembly, a phenomenon recently observed in HIV Env wherein certain gp41 antibodies can induce trimer disassembly (Turner et al., 2021). Here, we observe that non-trimer particles are more decorated with Fabs than the intact trimers consistent with some of the polyclonal Fabs being specific to these non-functional forms of Env. This is consistent with observations that polyclonal sera interact with non-functional forms of Env (Moore et al., 2006), the large discrepancy between ELISA and neutralization (Mason et al., 2016), and the similarity in the peptide fragment binding data between the neutralizing and non-neutralizing sera (Figure 3B). Of the small minority of stable Fabbound trimer classes we could reliably reconstruct in 3-D, we found that most nonneutralizing sera contained gp41 specificities similar to the HIV-1 non-nAb classes known to cause trimer disassembly (Turner et al., 2021). One dataset yielded a class with potential V1/N4 specificity similar to FZ019.2, however, it was a rare class with relatively few particles and likely represents a minor species within the larger polyclonal pool. Of the neutralizing sera, two of three yielded classes with clear glycan hole specificity and one with FZ019.2-like and gp41 specificity. No V1/V2-binding response was observed in EMPEM, possibly due either to low abundance of antibodies to this epitope region or disassembly of the SIVmac239 trimer by V1/N2-binding antibodies.

\section{Cryo-EM structure of SIVmac239 Env trimer in complex with the nAb K11}

To date there is only structural information available for the SIVmac239 delta V1/N2 gp120 monomer (Gorman et al., 2019) and no structure for a native-like SIVmac239 trimer. Our previous attempts to solve the trimer structure via cryo-EM failed due to severe orientation bias and trimer instability (von Bredow et al., 2019). However, by complexing the trimer with $\mathrm{K} 11 \mathrm{lgG}$, we were able to overcome these issues and obtain a $3.4 \AA$ - 
resolution reconstruction of the complex (Figures 4 and S4). Overall, the topology of the trimer closely resembles both the chimpanzee SIVcpz (MT145K), and HIV-1 Env (BG505), with the V2 and V3 loops forming the trimerization interface at the membrane-distal apex (Figures 4A and 4B). Alignment of the previously solved crystal structure with our cryoEM structure reveals a close fit in the gp120 core (root mean squared deviation $(R M S D)=0.67 \AA)$, but as expected, significant deviation around the gp41 and trimerization interfaces (full model RMSD=3.65 $\AA$; Figure S5A). The two features of the SIVmac Env structure that are most distinct from HIV and SIVcpz are the V4 loop and extended V1 loop which both fold back on top of the gp120 core (Figure 4B). These conformations are possible because of differences in glycosylation between SIVmac239 and both HIV and SIVcpz (Figure 4C). If we map the distinct SIVmac239 features on HIV and SIVcpz Env, the extended V1 loop would clash with the conserved HIV high-mannose patch glycans at positions N332 and N295 and the SIVcpz V4 glycan at N412, while the V4 loop would clash with the gp120 outer domain glycans at N363, N386, and N392 on both HIV and SIVcpz (Figure 4C), the latter two of which are highly conserved among HIV strains. To shield parts of the extended V1 loop, SIVmac239 has two glycans at positions N146 and N156 (Figures 4C and 4D). The majority of the extended V1 loop is well resolved in our cryo-EM map except for the stretch between residues 127 and 139, which appears to extend upward away from the gp120 core (Figure 4D). Overall, HIV BG505 SOSIP.664 is more similar to SIVcpz MT145K than SIVmac239, with RMSDs of $8.11 \AA$ and $9.79 \AA$ respectively (Figure S5B). Other regions of interest that show structural differences from SIVcpz and HIV are in the V5 loop, regions within gp41 HR1 and HR2, and the fusion peptide (FP; Figure S5C). The FP is buried against the gp120 core, unlike in HIV where it protrudes outwards, and SIVcpz where it is buried even further behind the fusion peptide proximal region helix (Figure S5C). SIVmac239 also features an additional disulfide bond between residues 194 and 206 in the V2 loop (Figures S5D).

Our cryo-EM structure confirms that K11 targets the same glycan hole as ITS90.03 (Figure 4E), which is located close to the well-characterized 241/289 glycan hole on BG505 SOSIP.664. In addition to the absence of a glycan sequon at position 254 , which has a PNGS in $20 \%$ of other SIVmac sequences (Gorman et al., 2019), another PNGS at N247 also sits within both the K11 and ITS90.03 epitopes and is almost completely unoccupied (Figures $4 \mathrm{~A}$ and $4 \mathrm{E}$ ). That both antibodies depend on this site being 
unoccupied for virus neutralization suggests the sub-occupancy is not unique to the soluble SOSIP trimer and must also occur on the native spike.

The K11 epitope-paratope interactions are largely dominated by sidechain-backbone hydrogen bonds between the CDRH3 loop and gp120 residues, as well as hydrogen bonds with the unique SIVmac239 glycan at N37, which interacts with both the $\mathrm{HC}$ and LC (Figure 4F). The K11 CDRH3 loop is 25 residues in length and features an inter-loop disulfide bond between residues $\mathrm{C} 100 \mathrm{c}$ and $\mathrm{C} 100_{\mathrm{K}}$ (Figure 4F). The glycans at positions N284, N295, and N371 also surround the epitope but are not engaged directly. Although the ITS90.03 and K11 epitopes overlap extensively (Figure 4E), the orientation of the two antibodies is orthogonal to one another, with K11 and ITS90.03 being oriented parallel and perpendicular to the viral membrane surface respectively (Figure 4F), which expands the binding footprint of ITS90.03 into the gp120-gp41 interface region.

\section{The glycans on SIVmac239 Env are more densely packed and shield more protein surface area than on HIV Env.}

Autologous $\mathrm{nAb}$ responses to SIVmac239 induced by infection are relatively weak compared to typical responses observed in HIV infection (Montefiori et al., 1996; Pedreño-Lopez et al., 2020; Richman et al., 2003; Wei et al., 2003; Wibmer et al., 2013). Since neutralization has been correlated with binding to native Env, we considered whether structural features of the SIVmac239 Env might provide clues as to a relatively low immunogenicity. Indeed, we found that the glycans on SIVmac239 Env are more closely packed and shield more protein surface than on HIV Env.

We determined the identity of all $\mathrm{N}$-linked glycans on Env via site-specific mass spectrometry (Figures S6A and S6B). The proportion of potential N-linked glycosylation sites (PNGS) containing high-mannose, complex-type, or no glycan at all are displayed above the sequence at each of the 27 PNGS (Figures 4A and 5A). Similar to HIV and SIVcpz Env trimers (Andrabi et al., 2019; Struwe et al., 2018), many of the sites contain oligomannose-type glycans (13 in total; greater than $80 \%$ of the total glycoforms observed) which indicate that, for the recombinant SIVmac239 Env trimer, these glycans are under steric constraints that prevent complete enzymatic processing. This can arise because of crowding from neighboring glycans or constraints from the surrounding protein structure 
(Berndsen et al., 2020). Six sites present a mixture of glycan processing states, both oligomannose and fully processed complex-type glycans, including two of the glycan sites located on gp41. Fully processed complex-type glycans are present on 8 sites on both gp120 and gp41 and these correspond to sites that are easily accessible to processing enzymes. This distribution of glycans is reminiscent of HIV and SIVcpz Env with two notable exceptions: the loss of gp120 outer-domain and high-mannose patch glycans due to the V1 and V4 loop conformations and the presence of a small fraction of glucosylated Man,GlcNAc2 (Man9) glycans.

The presence of glucosylated Man9 has important implications. Prior to the initial mannosidase trimming in the ER and Golgi, the Man9 glycan contains 1-3 glucose residues that act as a signal to the calnexin/calreticulin folding machinery that the protein has not folded correctly. Normally the enzyme that removes these glucose residues can readily access even those glycans that are restricted from further processing (Crispin et al., 2004) but glucosylated structures can still be secreted at sterically protected sites. Their presence could indicate that the glycan shield on SIVmac239 is even more densely packed than on either SIVcpz or HIV Env. There are two sites with detectable levels of monoglucosylated Man9, namely N37 and N278 but glucosylated structures can be secreted at sterically protected sites (Cutalo et al., 2004). Their presence could indicate that the glycan shield on SIVmac239 is even more densely packed than on either SIVcpz or HIV Env. There are two sites with detectable levels of monoglucosylated Man9, namely N37 and N278 (Cutalo et al., 2004) (equivalent to the N262 glycan on HIV when aligned with $\mathrm{HxB2}$ ), with the former having a higher percentage (Figure 5B). Both glycans are oriented more parallel to the gp120 protein surface, rather than perpendicular to it, and are engaging in stabilizing hydrogen bonds with surrounding protein residues (Figure 5B), which could restrict processing enzymes from accessing them, and suggests they are likely structurally important. Both sites are surrounded by several other glycans and N37 is even further stabilized by inter-glycan hydrogen bonds (Figure 5B). However, a caveat should be noted; because K11 also stabilizes the N37 glycan, we cannot say definitively if these interactions would be retained in the absence of the antibody.

To quantify the extent of glycan shielding across the trimer surface and the steric restriction of individual glycans, we used a slightly modified version of our previously published high-throughput atomistic modeling (HTAM) pipeline to generate a large 
ensemble of fully glycosylated SIVmac239 trimer structures (Berndsen et al., 2020; Chakraborty et al., 2020)(See Methods; Figures S6C and S6D). For comparison we also performed the same analysis on an equally sized ensemble of fully glycosylated BG505 SOSIP.664 models. By calculating the per-residue glycan encounter factor (GEF), a measure of glycan shielding, we found that overall SIVmac239 is more strongly shielded than BG505 despite having one less glycan per protomer (Figure 5C). The gp120 inner domain of SIVmac239 is the most strongly shielded while the glycan hole, V1, and V4 loops are the most exposed (excluding the trimer base; Figures 5C and 5D). As mentioned previously, the neutralizing antibody FZ019.2 epitope mapped to this region and docking of our cryo-EM structure into the nsEM map indicates that it's epitope likely contains segments of V1 as well as V4 (Figures 2C, 2D, S3C and S3D). Consistent with the higher level of shielding, the SIVmac239 glycans are more ordered overall (lower root mean squared fluctuation) (Figure 5D), which is indicative of more glycan-glycan interactions/crowding and/or glycan-protein interactions (Berndsen et al., 2020). The glycan at position N278 (262 in HxB2 numbering) has the lowest RMSF which is consistent with its high degree of ordering in the cryo-EM map and steric restriction implied by the presence of a Man9 at this site (Figure 5B). The glycan at N37, which contains an even higher percentage of glucosylated Man9, has a relatively average RMSF however, suggesting the restricted access of glycan processing enzymes is due in part to geometric constraints from its unique location and orientation in addition to glycan crowding or interactions with the protein surface.

\section{K11 protects rhesus macaques from repeated SIVmac239 challenge}

There are very few studies investigating the ability of antibody directly to protect against SIVmac239 infection (Fuchs et al., 2015; Gardner et al., 2019; Poignard et al., 2012). Although, not strictly an antibody, we showed that CD4-lgG2, a potent neutralizer of SIVmac239, protected against virus challenge at surprisingly low neutralizing titers (Poignard et al., 2012) compared to observations made for nAbs in the SHIV model or indeed in the AMP study (Corey et al., 2021; Pegu et al., 2019). One study did show protection in a single monkey against SIVmac239 challenge with the non-nAb $5 \mathrm{~L} 7$ at relatively high concentration (Fuchs et al., 2015). Accordingly, we investigated the protective efficacies of nAb K11 and non-nAb 5L7 against SIVmac239 in macaques. 
K11 was first shown to neutralize the replication competent SIVmac239 challenge stock at an $\mathrm{IC}_{50}$ of $2.2 \mu \mathrm{g} / \mathrm{mL}$, a 9 -fold lower potency than against the pseudovirus (Figure S7A). The challenge study consisted of three groups of rhesus macaques with six animals per group: Group 1: 5L7-LS group (LS mutation in the antibody Fc region to increase half-life (Ko et al., 2014)), Group 2: K11 group, and Group 3: untreated control group (Table S4). $\mathrm{K} 11$ or 5 L7-LS mAbs were infused by the intravenous route at a dose of $60 \mathrm{mg} / \mathrm{kg}$ one day prior to the challenge. All animals were subsequently challenged by the intravenous route with weekly repeat intravenous inoculation with $10^{5.5}$ AID50 of SIVmac239 (Fuchs et al., 2015) (Figure 6A). After three repeated weekly challenges, uninfected animals received a second $60 \mathrm{mg} / \mathrm{kg}$ dose of antibody, followed by repeated weekly virus challenges.

Following SIVmac239 challenge, 6/6 of control animals and 4/6 of 5L7-LS treated animals became infected after the first challenge (Figures 6B and $6 \mathrm{C}$ ). The remaining two animals r18011 and r18044 in the 5L7-LS group became infected 3 days after the second challenge. In contrast, all animals administered $\mathrm{K} 11$ showed protection after two challenges. Two K11-treated animals, r17041 and r18014 with the lowest serum concentrations (Figure S7B), became infected 7 days after the third challenge. Following the second infusion of K11 antibody, animals r18022 and rh2961 became infected after the seventh challenge and the remaining animal 17034 became infected after the eighth challenge (Figures 6B and 6C). Notably, animal r17075 in the K11 group was mistakenly detected as having detectable viral load after fourth challenge and was therefore not subjected to additional challenges. However, its viral load was undetectable since then, and the viral load repeat for the infection timepoint was negative. Therefore, this animal was excluded in the survival analysis shown in Figure 6B. None of the 5L7-LS-treated or $\mathrm{K} 11$-infected animals showed reduced peak viral load compared with the control group ( $P=0.219$ and $P=0.160$ respectively, Mann-Whitney test) (Figure 6D). Of note, $K 11$ significantly suppressed viral load during chronic phase infection $(P=0.004)$ compared with the control group while the non-nAb 5L7-LS antibody did not (Figure 6E).

Among the infected animals in the K11 group, the geometric mean of plasma SIVmac239 nAb ID 50 titers was 1:304 at 7 days prior to infection (Figures 6F and S7C), which is strikingly close to the number (1:299) from BG505 SOSIP vaccinated animals that were protected from SHIV BG505 $_{5}$ challenge (Pauthner et al., 2019). To investigate the 
contribution of effector function of antibody, we measured the antibody-dependent cellular cytotoxicity (ADCC) activity, where we observed better killing activity by SIVmac239specific mAbs than CD4-IgG2 (Figure 6G). However, the difference in ADCC activity between $\mathrm{K} 11$ and $5 \mathrm{~L} 7$ was not significant.

Taken together, our findings strongly indicate that, in the case of humoral immunity, antibody neutralization activity is necessary and sufficient for protection against SIVmac239 virus challenge.

\section{DISCUSSION}

The phase Ilb HVTN703/HPTN081 and HVTN704/HPTN085 trials (also known as the AMP study) showed that a bnAb can protect humans against HIV exposure, albeit at relatively high serum antibody neutralizing titers (Corey et al., 2021). Many studies show that bnAbs can protect NHPs against SHIV challenge, again at similar relatively high serum antibody titers (Pegu et al., 2019). T cell responses have clinically failed to protect humans against HIV exposure in a variety of formats (Buchbinder et al., 2008; Cohen, 2021; Gray et al., 2011; Hammer et al., 2013) but, CMV vectors that induce MHC-Erestricted $\mathrm{T}$ cell responses have provided substantial control of viral replication after SIVmac239 challenge (Hansen et al., 2011, 2013, 2019). There is considerable motivation to determine whether combining antibody and $\mathrm{T}$ cell responses can enhance protection. This investigation would be most readily conducted in NHPs but that requires deploying both arms of immunity against either SHIV or SIV. Given that SIV is considered a more robust model of HIV, we have focused on generating the appropriate humoral reagents, namely SIV-specific neutralizing antibodies and an SIV trimer immunogen. Here, we described the generation and characterization of both.

We isolated 12 nAbs against SIVmac239, characterized one (K11) in some detail, showed that it binds to a glycan hole on gp120 and that it can protect macaques against SIVmac239 challenge. The serum nAb titers required for protection were comparable to those noted for protection against HIV in humans and SHIV in macaques (Pauthner et al., 2019; Pegu et al., 2019). Thus, at least from a nAb standpoint, it does not appear that SIVmac239 is more resistant per se to neutralization or requires higher nAb titers for protection than HIV or SHIV. Rather, in infected macaques the virus tends to elicit nAb 
responses that are of low neutralization potency, rare, or delayed compared with many HIV and SHIV infections, reflecting the poor immunogenicity of nAb epitopes on SIVmac239 Env. Notwithstanding, the nAb K11 is now available as a passive reagent to be combined with $\mathrm{T}$ cell responses to investigate synergy of humoral and cellular protection against the rigorous SIVmac239 challenge.

We generated a stabilized recombinant trimer of SIVmac239 and solved its structure in complex with nAb K11 by cryoEM. This is the first high-resolution structure of a soluble macaque SIV trimer. The trimer was shown to closely resemble trimers of HIV and of chimpanzee SIV but with distinct features, notably a V4 loop and an extended V1 loop that fold back on top of the gp120 core; conformations made possible by differences in the glycan shield relative to HIV and SIVcpz. Although SIVmac239 contains two additional glycans on its extended V1 loop, our computational modeling revealed it is still one of the poorly shielded regions of the trimer. This is consistent with the prevalence of antibodies targeting this region such as FZ019.2. The absence of antibodies targeting the rest of the highly exposed $\mathrm{V} 1$ loop in all the polyclonal sera tested is perplexing, especially relative to the abundance of V4/V5-targeting antibodies, seeing how V5 is significantly more shielded by glycans. The functional significance of this exposed extended V1 loop is unclear, though it could be acting to protect the more conserved gp120 core which is shielded by glycans on SIVcpz and HIV Env. A HTAM pipeline was used to generate a large ensemble of fully glycosylated SIVmac239 trimer structures and show that the SIVmac239 trimer more densely packed N-linked glycans than the prototypic BG505 HIV trimer. This higher shielding may be associated with the relatively low levels of $n A b$ responses to the SIVmac239 trimer in natural infection (Pedreño-Lopez et al., 2020). Further, the abundance of antibody responses to degraded forms of Env, likely related to the poor stability of the trimer, may also play a role. The availability of the recombinant trimer will now however permit exploration of the molecular origins of this low immunogenicity in macaques. Additionally, the high-resolution structure also enables rational engineering of the trimer to improve stability and potentially immunogenicity. Indeed, previous studies suggest that removal of one or more glycans from SIVmac239 Env can enhance $\mathrm{nAb}$ responses to wild-type virus induced by infection with the glycandeficient virus (Reitter and Desrosiers, 1998; Reitter et al., 1998). 
Overall, our studies represent a notable advance in our understanding of the interaction of the humoral immune system with the prototypic SIVmac239 and pave the way for exploring synergies between humoral and cellular immune systems in the SIV model that will directly inform HIV vaccine design strategies.

\section{ACKNOWLEDGEMENT}

We thank Rosemarie Mason and Mario Roederer at NIH, David Montefiori at Duke University, and Cynthia Derdeyn at Emory University for providing the SIV pseudovirus envelope plasmids. We thank Louis Picker at Oregon Health \& Science University for providing the non-neutralizing SIVmac239 infected plasma samples. We thank Matthias Pauthner for testing the plasma nAb titers from previous infected rhesus samples and the staff of the Quantitative Molecular Diagnostics Core of the AIDS and Cancer Virus Program, Frederick National Laboratory for technical assistance with viral load measurements. This work is supported by National Institute of Allergy and Infectious Diseases (NIAID) Consortium for HIVIAIDS Vaccine Development (CHAVD; UM1Al144462) (M.C., A.B.W., D.R.B. and D.S.) and the Bill and Melinda Gates Foundation through the Collaboration for AIDS Vaccine Discovery (INV008352/OPP1153692 and OPP1196345/INV-008813)(D.S., D.R.B., A.B.W., and R.A.); in part by NIH Grant RO1 AI 52056-20 (D.I.W.), and Federal funds from the National Cancer Institute, National Institutes of Health, under Contract Nos. HHSN261200800001E and 75N91019D00024 (J.D.L.). This work is also supported in part by the Office of The Director, National Institutes of Health under Award Number P510D011106 to the Wisconsin National Primate Research Center, University of Wisconsin-Madison. This research was conducted in part at a facility constructed with support from Research Facilities Improvement Program grant numbers RR15459-01 and RR020141-01.

\section{AUTHOR CONTRIBUTIONS}

F.Z., D.R.B., and D.S. conceived and designed the study. F.Z. and A.B. performed sorting, antibody cloning and sequencing experiments. F.Z., A.B., S.B., and O.L. expressed and purified the monoclonal antibodies. N.P., J.L., T.V., F.L., B.R., and M.R. expressed and purified 5L7-LS antibody. F.Z., A.B., and S.B. characterized monoclonal antibodies in functional assays. F.Z., A.B. expressed the recombinant SIVmac239 gp120 delta V1V2, delta V3 and gp140 FT proteins. G.S., P.Y., and R.A. expressed the recombinant SIVmac239 SOSIP.664 protein for Cryo-EM and nsEM. F.Z., A.B., and S.B. produced 
pseudovirus and performed TZM-bl assay. Z.T.B performed Cryo-EM experiments, sample preparation, imaging, data processing and model building. Z.T.B., W.L., L.S., and G.O. performed nsEM experiments and Z.T.B and W.L. processed the data. J.D.A. performed LC-MS analysis; J.D.A. and M.C. analyzed and interpreted data. R.C.D. provided the SIVmac239 challenge stock. R.N. provided the neutralization data against challenge stock and the ADCC data. E.G.R., D.B., N.P., R.C.D. and D.I.W. planned the animal challenge studies. K.L.W. and E.G.R. performed the antibody infusion and SIVmac239 challenge study. K.L.W. and E.G.R. collected the plasma samples, and J.D.L. measured viral load. N.P. and D.I.W. analyzed peak viral load and measured rhesus serum antibody concentrations. S.C. performed computational modeling of the glycan shield and S.C. and Z.T.B. analyzed and interpreted the modeling data. F.Z., Z.T.B., A.B.W., D.R.B. and D.S. wrote the manuscript and all authors reviewed and edited the manuscript.

\section{DECLARATION OF INTERESTS}

The authors declare no competing interests.

\section{REFERENCES}

Agirre, J., Iglesias-Fernández, J., Rovira, C., Davies, G.J., Wilson, K.S., and Cowtan, K.D. (2015). Privateer: software for the conformational validation of carbohydrate structures. Nat. Struct. Mol. Biol. 22, 833-834.

Alpert, M.D., Heyer, L.N., Williams, D.E.J., Harvey, J.D., Greenough, T., Allhorn, M., and Evans, D.T. (2012). A novel assay for antibody-dependent cell-mediated cytotoxicity against HIV-1- or SIV-infected cells reveals incomplete overlap with antibodies measured by neutralization and binding assays. J. Virol. 86, 12039-12052. Andrabi, R., Pallesen, J., Allen, J.D., Song, G., Zhang, J., de Val, N., Gegg, G., Porter, K., Su, C.-Y., Pauthner, M., et al. (2019). The chimpanzee SIV envelope trimer: structure and deployment as an HIV vaccine template. Cell Rep. 27, 2426-2441.e6. Arunachalam, P.S., Charles, T.P., Joag, V., Bollimpelli, V.S., Scott, M.K.D., Wimmers, F., Burton, S.L., Labranche, C.C., Petitdemange, C., Gangadhara, S., et al. (2020). T cell-inducing vaccine durably prevents mucosal SHIV infection even with lower neutralizing antibody titers. Nat. Med. 26, 932-940.

Asmal, M., Luedemann, C., Lavine, C.L., Mach, L.V., Balachandran, H., Brinkley, C., Denny, T.N., Lewis, M.G., Anderson, H., Pal, R., et al. (2015). Infection of monkeys by 
simian-human immunodeficiency viruses with transmitted/founder clade C HIV-1 envelopes. Virology 475, 37-45.

Barad, B.A., Echols, N., Wang, R.Y.-R., Cheng, Y., DiMaio, F., Adams, P.D., and Fraser, J.S. (2015). EMRinger: side chain-directed model and map validation for 3D cryo-electron microscopy. Nat. Methods 12, 943-946.

Barouch, D.H., Liu, J., Li, H., Maxfield, L.F., Abbink, P., Lynch, D.M., lampietro, M.J., SanMiguel, A., Seaman, M.S., Ferrari, G., et al. (2012). Vaccine protection against acquisition of neutralization-resistant SIV challenges in rhesus monkeys. Nature 482 , 89-93.

Berndsen, Z.T., Chakraborty, S., Wang, X., Cottrell, C.A., Torres, J.L., Diedrich, J.K., López, C.A., Yates, J.R., van Gils, M.J., Paulson, J.C., et al. (2020). Visualization of the HIV-1 Env glycan shield across scales. Proc. Natl. Acad. Sci. USA 117, 28014-28025. Bianchi, M., Turner, H.L., Nogal, B., Cottrell, C.A., Oyen, D., Pauthner, M., Bastidas, R., Nedellec, R., McCoy, L.E., Wilson, I.A., et al. (2018). Electron-Microscopy-Based Epitope Mapping Defines Specificities of Polyclonal Antibodies Elicited during HIV-1 BG505 Envelope Trimer Immunization. Immunity 49, 288-300.e8.

von Bredow, B., Andrabi, R., Grunst, M., Grandea, A.G., Le, K., Song, G., Berndsen, Z.T., Porter, K., Pallesen, J., Ward, A.B., et al. (2019). Differences in the Binding Affinity of an HIV-1 V2 Apex-Specific Antibody for the SIVsmm/mac Envelope Glycoprotein Uncouple Antibody-Dependent Cellular Cytotoxicity from Neutralization. MBio 10, e01255-19.

Buchbinder, S.P., Mehrotra, D.V., Duerr, A., Fitzgerald, D.W., Mogg, R., Li, D., Gilbert, P.B., Lama, J.R., Marmor, M., Del Rio, C., et al. (2008). Efficacy assessment of a cellmediated immunity HIV-1 vaccine (the Step Study): a double-blind, randomised, placebo-controlled, test-of-concept trial. Lancet 372, 1881-1893.

Casimiro, D.R., Wang, F., Schleif, W.A., Liang, X., Zhang, Z.-Q., Tobery, T.W., Davies, M.-E., McDermott, A.B., O’Connor, D.H., Fridman, A., et al. (2005). Attenuation of simian immunodeficiency virus SIVmac239 infection by prophylactic immunization with dna and recombinant adenoviral vaccine vectors expressing Gag. J. Virol. 79, 1554715555.

Chakraborty, S., Berndsen, Z.T., Hengartner, N.W., Korber, B.T., Ward, A.B., and Gnanakaran, S. (2020). Quantification of the Resilience and Vulnerability of HIV-1 Native Glycan Shield at Atomistic Detail. IScience 23, 101836.

Chanzu, N., and Ondondo, B. (2014). Induction of Potent and Long-Lived Antibody and 
Cellular Immune Responses in the Genitorectal Mucosa Could be the Critical Determinant of HIV Vaccine Efficacy. Front. Immunol. 5, 202.

Chen, V.B., Arendall, W.B., Headd, J.J., Keedy, D.A., Immormino, R.M., Kapral, G.J., Murray, L.W., Richardson, J.S., and Richardson, D.C. (2010). MolProbity: all-atom structure validation for macromolecular crystallography. Acta Crystallogr. Sect. D, Biol. Crystallogr. 66, 12-21.

Cirelli, K.M., Carnathan, D.G., Nogal, B., Martin, J.T., Rodriguez, O.L., Upadhyay, A.A., Enemuo, C.A., Gebru, E.H., Choe, Y., Viviano, F., et al. (2019). Slow delivery immunization enhances HIV neutralizing antibody and germinal center responses via modulation of immunodominance. Cell 177, 1153-1171.e28.

Cohen, J. (2021). https://www.science.org/content/article/failed-hiv-vaccine-trial-marksanother-setback-field.

Corey, L., Gilbert, P.B., Juraska, M., Montefiori, D.C., Morris, L., Karuna, S.T., Edupuganti, S., Mgodi, N.M., deCamp, A.C., Rudnicki, E., et al. (2021). Two Randomized Trials of Neutralizing Antibodies to Prevent HIV-1 Acquisition. N. Engl. J. Med. 384, 1003-1014.

Cottrell, C.A., van Schooten, J., Bowman, C.A., Yuan, M., Oyen, D., Shin, M., Morpurgo, R., van der Woude, P., van Breemen, M., Torres, J.L., et al. (2020). Mapping the immunogenic landscape of near-native HIV-1 envelope trimers in non-human primates. PLoS Pathog. 16, e1008753.

Crispin, M.D.M., Ritchie, G.E., Critchley, A.J., Morgan, B.P., Wilson, I.A., Dwek, R.A., Sim, R.B., and Rudd, P.M. (2004). Monoglucosylated glycans in the secreted human complement component C3: implications for protein biosynthesis and structure. FEBS Lett. 566, 270-274.

Cutalo, J.M., Deterding, L.J., and Tomer, K.B. (2004). Characterization of glycopeptides from HIV-I(SF2) gp120 by liquid chromatography mass spectrometry. J Am Soc Mass Spectrom 15, 1545-1555.

Daniel, M.D., Letvin, N.L., King, N.W., Kannagi, M., Sehgal, P.K., Hunt, R.D., Kanki, P.J., Essex, M., and Desrosiers, R.C. (1985). Isolation of T-cell tropic HTLV-III-like retrovirus from macaques. Science 228, 1201-1204.

Daniel, M.D., Kirchhoff, F., Czajak, S.C., Sehgal, P.K., and Desrosiers, R.C. (1992). Protective effects of a live attenuated SIV vaccine with a deletion in the nef gene.

Science 258, 1938-1941.

Del Prete, G.Q., Ailers, B., Moldt, B., Keele, B.F., Estes, J.D., Rodriguez, A., Sampias, 
M., Oswald, K., Fast, R., Trubey, C.M., et al. (2014). Selection of unadapted, pathogenic SHIVs encoding newly transmitted HIV-1 envelope proteins. Cell Host Microbe 16, 412-418.

Doores, K.J., and Burton, D.R. (2010). Variable loop glycan dependency of the broad and potent HIV-1-neutralizing antibodies PG9 and PG16. J. Virol. 84, 10510-10521. Dunbar, J., Krawczyk, K., Leem, J., Marks, C., Nowak, J., Regep, C., Georges, G., Kelm, S., Popovic, B., and Deane, C.M. (2016). SAbPred: a structure-based antibody prediction server. Nucleic Acids Res. 44, W474-8.

Emsley, P., and Crispin, M. (2018). Structural analysis of glycoproteins: building Nlinked glycans with Coot. Acta Crystallogr. D Struct. Biol. 74, 256-263.

Francica, J.R., Sheng, Z., Zhang, Z., Nishimura, Y., Shingai, M., Ramesh, A., Keele, B.F., Schmidt, S.D., Flynn, B.J., Darko, S., et al. (2015). Analysis of immunoglobulin transcripts and hypermutation following SHIV(AD8) infection and protein-plus-adjuvant immunization. Nat. Commun. 6, 6565.

Fuchs, S.P., Martinez-Navio, J.M., Piatak, M., Lifson, J.D., Gao, G., and Desrosiers, R.C. (2015). AAV-Delivered Antibody Mediates Significant Protective Effects against SIVmac239 Challenge in the Absence of Neutralizing Activity. PLoS Pathog. 11, e1005090.

Gardner, M.R., Fellinger, C.H., Kattenhorn, L.M., Davis-Gardner, M.E., Weber, J.A., Alfant, B., Zhou, A.S., Prasad, N.R., Kondur, H.R., Newton, W.A., et al. (2019). AAVdelivered eCD4-Ig protects rhesus macaques from high-dose SIVmac239 challenges. Sci. Transl. Med. 11, eaau5409.

Gorman, J., Mason, R.D., Nettey, L., Cavett, N., Chuang, G.-Y., Peng, D., Tsybovsky, Y., Verardi, R., Nguyen, R., Ambrozak, D., et al. (2019). Isolation and Structure of an Antibody that Fully Neutralizes Isolate SIVmac239 Reveals Functional Similarity of SIV and HIV Glycan Shields. Immunity 51, 724-734.e4.

Gray, G.E., Allen, M., Moodie, Z., Churchyard, G., Bekker, L.-G., Nchabeleng, M., Mlisana, K., Metch, B., de Bruyn, G., Latka, M.H., et al. (2011). Safety and efficacy of the HVTN 503/Phambili study of a clade-B-based HIV-1 vaccine in South Africa: a double-blind, randomised, placebo-controlled test-of-concept phase $2 \mathrm{~b}$ study. Lancet Infect. Dis. 11, 507-515.

Hammer, S.M., Sobieszczyk, M.E., Janes, H., Karuna, S.T., Mulligan, M.J., Grove, D., Koblin, B.A., Buchbinder, S.P., Keefer, M.C., Tomaras, G.D., et al. (2013). Efficacy trial of a DNA/rAd5 HIV-1 preventive vaccine. N. Engl. J. Med. 369, 2083-2092. 
Hansen, S.G., Ford, J.C., Lewis, M.S., Ventura, A.B., Hughes, C.M., Coyne-Johnson, L., Whizin, N., Oswald, K., Shoemaker, R., Swanson, T., et al. (2011). Profound early control of highly pathogenic SIV by an effector memory T-cell vaccine. Nature $473,523-$ 527.

Hansen, S.G., Piatak, M., Ventura, A.B., Hughes, C.M., Gilbride, R.M., Ford, J.C., Oswald, K., Shoemaker, R., Li, Y., Lewis, M.S., et al. (2013). Immune clearance of highly pathogenic SIV infection. Nature 502, 100-104.

Hansen, S.G., Marshall, E.E., Malouli, D., Ventura, A.B., Hughes, C.M., Ainslie, E., Ford, J.C., Morrow, D., Gilbride, R.M., Bae, J.Y., et al. (2019). A live-attenuated RhCMVISIV vaccine shows long-term efficacy against heterologous SIV challenge. Sci. Transl. Med. 11, eaaw2607.

Harouse, J.M., Gettie, A., Tan, R.C., Blanchard, J., and Cheng-Mayer, C. (1999).

Distinct pathogenic sequela in rhesus macaques infected with CCR5 or CXCR4 utilizing SHIVs. Science 284, 816-819.

Herzik, M.A., Fraser, J.S., and Lander, G.C. (2019). A Multi-model Approach to Assessing Local and Global Cryo-EM Map Quality. Structure 27, 344-358.e3. Huang, J., Doria-Rose, N.A., Longo, N.S., Laub, L., Lin, C.-L., Turk, E., Kang, B.H., Migueles, S.A., Bailer, R.T., Mascola, J.R., et al. (2013). Isolation of human monoclonal antibodies from peripheral blood B cells. Nat. Protoc. 8, 1907-1915.

Julg, B., Sok, D., Schmidt, S.D., Abbink, P., Newman, R.M., Broge, T., Linde, C., Nkolola, J., Le, K., Su, D., et al. (2017). Protective Efficacy of Broadly Neutralizing Antibodies with Incomplete Neutralization Activity Against SHIV in Rhesus Monkeys. J. Virol. 91, e01187-17.

Kilgore, K.M., Murphy, M.K., Burton, S.L., Wetzel, K.S., Smith, S.A., Xiao, P., Reddy, S., Francella, N., Sodora, D.L., Silvestri, G., et al. (2015). Characterization and implementation of a diverse simian immunodeficiency virus sivsm envelope panel in the assessment of neutralizing antibody breadth elicited in rhesus macaques by multimodal vaccines expressing the sivmac239 envelope. J. Virol. 89, 8130-8151.

Ko, S.-Y., Pegu, A., Rudicell, R.S., Yang, Z., Joyce, M.G., Chen, X., Wang, K., Bao, S., Kraemer, T.D., Rath, T., et al. (2014). Enhanced neonatal Fc receptor function improves protection against primate SHIV infection. Nature 514, 642-645.

Li, H., Wang, S., Kong, R., Ding, W., Lee, F.-H., Parker, Z., Kim, E., Learn, G.H., Hahn, P., Policicchio, B., et al. (2016). Envelope residue 375 substitutions in simian-human immunodeficiency viruses enhance CD4 binding and replication in rhesus macaques. 
Proc. Natl. Acad. Sci. USA 113, E3413-22.

Martins, M.A., Bischof, G.F., Shin, Y.C., Lauer, W.A., Gonzalez-Nieto, L., Watkins, D.I., Rakasz, E.G., Lifson, J.D., and Desrosiers, R.C. (2019). Vaccine protection against SIVmac239 acquisition. Proc. Natl. Acad. Sci. USA 116, 1739-1744.

Mason, R.D., Welles, H.C., Adams, C., Chakrabarti, B.K., Gorman, J., Zhou, T., Nguyen, R., O’Dell, S., Lusvarghi, S., Bewley, C.A., et al. (2016). Targeted Isolation of Antibodies Directed against Major Sites of SIV Env Vulnerability. PLoS Pathog. 12, e1005537.

McMichael, A.J., and Koff, W.C. (2014). Vaccines that stimulate T cell immunity to HIV1: the next step. Nat. Immunol. 15, 319-322.

Montefiori, D.C., Baba, T.W., Li, A., Bilska, M., and Ruprecht, R.M. (1996). Neutralizing and infection-enhancing antibody responses do not correlate with the differential pathogenicity of SIVmac239delta3 in adult and infant rhesus monkeys. J. Immunol. 157, 5528-5535.

Moore, P.L., Crooks, E.T., Porter, L., Zhu, P., Cayanan, C.S., Grise, H., Corcoran, P., Zwick, M.B., Franti, M., Morris, L., et al. (2006). Nature of nonfunctional envelope proteins on the surface of human immunodeficiency virus type 1. J. Virol. 80, 25152528.

Pauthner, M.G., Nkolola, J.P., Havenar-Daughton, C., Murrell, B., Reiss, S.M., Bastidas, R., Prévost, J., Nedellec, R., von Bredow, B., Abbink, P., et al. (2019). Vaccine-Induced Protection from Homologous Tier 2 SHIV Challenge in Nonhuman Primates Depends on Serum-Neutralizing Antibody Titers. Immunity 50, 241-252.e6.

Pedreño-Lopez, N., Dang, C.M., Rosen, B.C., Ricciardi, M.J., Bailey, V.K., Gutman, M.J., Gonzalez-Nieto, L., Pauthner, M.G., Le, K., Song, G., et al. (2020). Induction of Transient Virus Replication Facilitates Antigen-Independent Isolation of SIV-Specific Monoclonal Antibodies. Mol. Ther. Methods Clin. Dev. 16, 225-237.

Pegu, A., Yang, Z., Boyington, J.C., Wu, L., Ko, S.-Y., Schmidt, S.D., McKee, K., Kong, W.-P., Shi, W., Chen, X., et al. (2014). Neutralizing antibodies to HIV-1 envelope protect more effectively in vivo than those to the CD4 receptor. Sci. Transl. Med. 6, 243 ra88. Pegu, A., Borate, B., Huang, Y., Pauthner, M.G., Hessell, A.J., Julg, B., Doria-Rose, N.A., Schmidt, S.D., Carpp, L.N., Cully, M.D., et al. (2019). A Meta-analysis of Passive Immunization Studies Shows that Serum-Neutralizing Antibody Titer Associates with Protection against SHIV Challenge. Cell Host Microbe 26, 336-346.e3.

Pettersen, E.F., Goddard, T.D., Huang, C.C., Couch, G.S., Greenblatt, D.M., Meng, 
E.C., and Ferrin, T.E. (2004). UCSF Chimera - a visualization system for exploratory research and analysis. J. Comput. Chem. 25, 1605-1612.

Pettersen, E.F., Goddard, T.D., Huang, C.C., Meng, E.C., Couch, G.S., Croll, T.I., Morris, J.H., and Ferrin, T.E. (2021). UCSF ChimeraX: Structure visualization for researchers, educators, and developers. Protein Sci. 30, 70-82.

Pintilie, G., and Chiu, W. (2012). Comparison of Segger and other methods for segmentation and rigid-body docking of molecular components in cryo-EM density maps. Biopolymers 97, 742-760.

Pintilie, G.D., Zhang, J., Goddard, T.D., Chiu, W., and Gossard, D.C. (2010).

Quantitative analysis of cryo-EM density map segmentation by watershed and scalespace filtering, and fitting of structures by alignment to regions. J. Struct. Biol. 170, 427438.

Poignard, P., Moldt, B., Maloveste, K., Campos, N., Olson, W.C., Rakasz, E., Watkins, D.I., and Burton, D.R. (2012). Protection against high-dose highly pathogenic mucosal SIV challenge at very low serum neutralizing titers of the antibody-like molecule CD4IgG2. PLoS One 7, e42209.

Punjani, A., and Fleet, D.J. (2021). 3D variability analysis: Resolving continuous flexibility and discrete heterogeneity from single particle cryo-EM. J. Struct. Biol. 213, 107702.

Punjani, A., Rubinstein, J.L., Fleet, D.J., and Brubaker, M.A. (2017). cryoSPARC: algorithms for rapid unsupervised cryo-EM structure determination. Nat. Methods 14, 290-296.

Reitter, J.N., and Desrosiers, R.C. (1998). Identification of replication-competent strains of simian immunodeficiency virus lacking multiple attachment sites for $\mathrm{N}$-linked carbohydrates in variable regions 1 and 2 of the surface envelope protein. J. Virol. 72, 5399-5407.

Reitter, J.N., Means, R.E., and Desrosiers, R.C. (1998). A role for carbohydrates in immune evasion in AIDS. Nat. Med. 4, 679-684.

Reynolds, M.R., Weiler, A.M., Weisgrau, K.L., Piaskowski, S.M., Furlott, J.R., Weinfurter, J.T., Kaizu, M., Soma, T., León, E.J., MacNair, C., et al. (2008). Macaques vaccinated with live-attenuated SIV control replication of heterologous virus. J. Exp. Med. 205, 2537-2550.

Richman, D.D., Wrin, T., Little, S.J., and Petropoulos, C.J. (2003). Rapid evolution of the neutralizing antibody response to HIV type 1 infection. Proc. Natl. Acad. Sci. USA 
100, 4144-4149.

Roederer, M., Keele, B.F., Schmidt, S.D., Mason, R.D., Welles, H.C., Fischer, W., Labranche, C., Foulds, K.E., Louder, M.K., Yang, Z.-Y., et al. (2014). Immunological and virological mechanisms of vaccine-mediated protection against SIV and HIV. Nature 505, 502-508.

Shingai, M., Donau, O.K., Plishka, R.J., Buckler-White, A., Mascola, J.R., Nabel, G.J., Nason, M.C., Montefiori, D., Moldt, B., Poignard, P., et al. (2014). Passive transfer of modest titers of potent and broadly neutralizing anti-HIV monoclonal antibodies block SHIV infection in macaques. J. Exp. Med. 211, 2061-2074.

Sok, D., Laserson, U., Laserson, J., Liu, Y., Vigneault, F., Julien, J.-P., Briney, B., Ramos, A., Saye, K.F., Le, K., et al. (2013). The effects of somatic hypermutation on neutralization and binding in the PGT121 family of broadly neutralizing HIV antibodies. PLoS Pathog. 9, e1003754.

Struwe, W.B., Chertova, E., Allen, J.D., Seabright, G.E., Watanabe, Y., Harvey, D.J., Medina-Ramirez, M., Roser, J.D., Smith, R., Westcott, D., et al. (2018). Site-Specific Glycosylation of Virion-Derived HIV-1 Env Is Mimicked by a Soluble Trimeric Immunogen. Cell Rep. 24, 1958-1966.e5.

Suloway, C., Pulokas, J., Fellmann, D., Cheng, A., Guerra, F., Quispe, J., Stagg, S., Potter, C.S., and Carragher, B. (2005). Automated molecular microscopy: the new Leginon system. J. Struct. Biol. 151, 41-60.

Sundling, C., Li, Y., Huynh, N., Poulsen, C., Wilson, R., O’Dell, S., Feng, Y., Mascola, J.R., Wyatt, R.T., and Karlsson Hedestam, G.B. (2012). High-resolution definition of vaccine-elicited $B$ cell responses against the HIV primary receptor binding site. Sci. Transl. Med. 4, 142 ra96.

Tomaras, G.D., Yates, N.L., Liu, P., Qin, L., Fouda, G.G., Chavez, L.L., Decamp, A.C., Parks, R.J., Ashley, V.C., Lucas, J.T., et al. (2008). Initial B-cell responses to transmitted human immunodeficiency virus type 1: virion-binding immunoglobulin $\mathrm{M}$ (IgM) and IgG antibodies followed by plasma anti-gp41 antibodies with ineffective control of initial viremia. J. Virol. 82, 12449-12463.

Turner, H.L., Andrabi, R., Cottrell, C.A., Richey, S.T., Song, G., Callaghan, S., Anzanello, F., Moyer, T.J., Abraham, W., Melo, M., et al. (2021). Disassembly of HIV envelope glycoprotein trimer immunogens is driven by antibodies elicited via immunization. Sci. Adv. 7, eabh2791.

Walker, L.M., Huber, M., Doores, K.J., Falkowska, E., Pejchal, R., Julien, J.-P., Wang, 
S.-K., Ramos, A., Chan-Hui, P.-Y., Moyle, M., et al. (2011). Broad neutralization coverage of HIV by multiple highly potent antibodies. Nature 477, 466-470.

Wang, R.Y.-R., Song, Y., Barad, B.A., Cheng, Y., Fraser, J.S., and DiMaio, F. (2016). Automated structure refinement of macromolecular assemblies from cryo-EM maps using Rosetta. Elife 5, e17219.

Waterhouse, A., Bertoni, M., Bienert, S., Studer, G., Tauriello, G., Gumienny, R., Heer, F.T., de Beer, T.A.P., Rempfer, C., Bordoli, L., et al. (2018). SWISS-MODEL: homology modelling of protein structures and complexes. Nucleic Acids Res. 46, W296-W303. Watkins, D.I., Burton, D.R., Kallas, E.G., Moore, J.P., and Koff, W.C. (2008). Nonhuman primate models and the failure of the Merck HIV-1 vaccine in humans. Nat. Med. 14, 617-621.

Wei, X., Decker, J.M., Wang, S., Hui, H., Kappes, J.C., Wu, X., Salazar-Gonzalez, J.F., Salazar, M.G., Kilby, J.M., Saag, M.S., et al. (2003). Antibody neutralization and escape by HIV-1. Nature 422, 307-312.

Wibmer, C.K., Bhiman, J.N., Gray, E.S., Tumba, N., Abdool Karim, S.S., Williamson, C., Morris, L., and Moore, P.L. (2013). Viral escape from HIV-1 neutralizing antibodies drives increased plasma neutralization breadth through sequential recognition of multiple epitopes and immunotypes. PLoS Pathog. 9, e1003738.

Wilson, N.A., Reed, J., Napoe, G.S., Piaskowski, S., Szymanski, A., Furlott, J., Gonzalez, E.J., Yant, L.J., Maness, N.J., May, G.E., et al. (2006). Vaccine-induced cellular immune responses reduce plasma viral concentrations after repeated low-dose challenge with pathogenic simian immunodeficiency virus SIVmac239. J. Virol. 80, 5875-5885.

Wu, X., Yang, Z.-Y., Li, Y., Hogerkorp, C.-M., Schief, W.R., Seaman, M.S., Zhou, T., Schmidt, S.D., Wu, L., Xu, L., et al. (2010). Rational design of envelope identifies broadly neutralizing human monoclonal antibodies to HIV-1. Science 329, 856-861. Zhang, K. (2016). Gctf: Real-time CTF determination and correction. J. Struct. Biol. 193, 1-12.

Zhao, F., Joyce, C., Burns, A., Nogal, B., Cottrell, C.A., Ramos, A., Biddle, T., Pauthner, M., Nedellec, R., Qureshi, H., et al. (2020). Mapping neutralizing antibody epitope specificities to an HIV env trimer in immunized and in infected rhesus macaques. Cell Rep. 32, 108122.

Zheng, S.Q., Palovcak, E., Armache, J.-P., Verba, K.A., Cheng, Y., and Agard, D.A. (2017). MotionCor2: anisotropic correction of beam-induced motion for improved cryo- 
electron microscopy. Nat. Methods 14, 331-332.

Zivanov, J., Nakane, T., Forsberg, B.O., Kimanius, D., Hagen, W.J., Lindahl, E., and Scheres, S.H. (2018). New tools for automated high-resolution cryo-EM structure determination in RELION-3. Elife 7, e42166. 


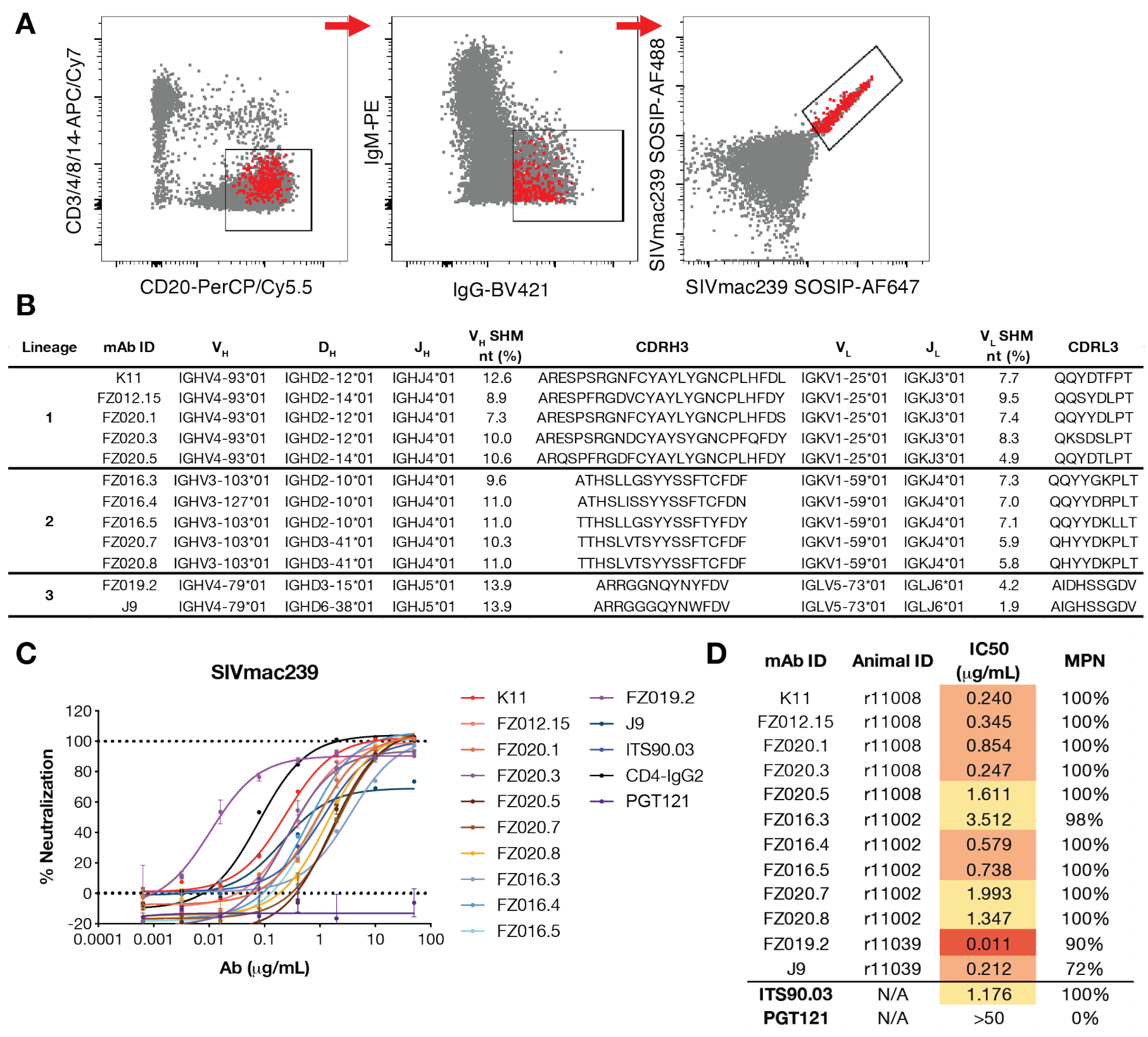

Figure 1. Isolation of SIVmac239 neutralizing rhesus mAbs. (A) FACS sorting layout from r10039 rhesus PBMCs, in which population is gated on lymphocytes/singlets. CD3-CD4-CD8-CD14-CD20+'IgMIgG+ SIVmac239 SOSIP.664 ${ }^{2+}$ memory B cells were single-cell sorted. Red dots represent sorted cells. (B) Immunogenetics of isolated rhesus mAbs. Rhesus mAbs were annotated with a rhesus germline database (Cirelli et al., 2019). (C-D) SIVmac239 pseudovirus neutralization curves (C) and summary table (D) of neutralization $\mathrm{IC}_{50}$ and maximum percentage of neutralization (MPN) for denoted mAbs. 
A

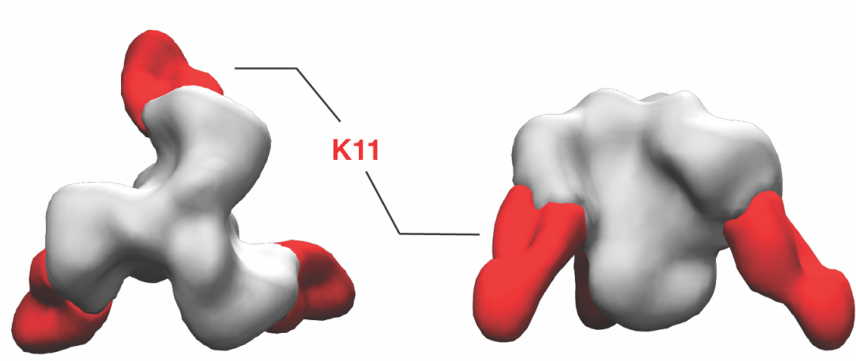

C

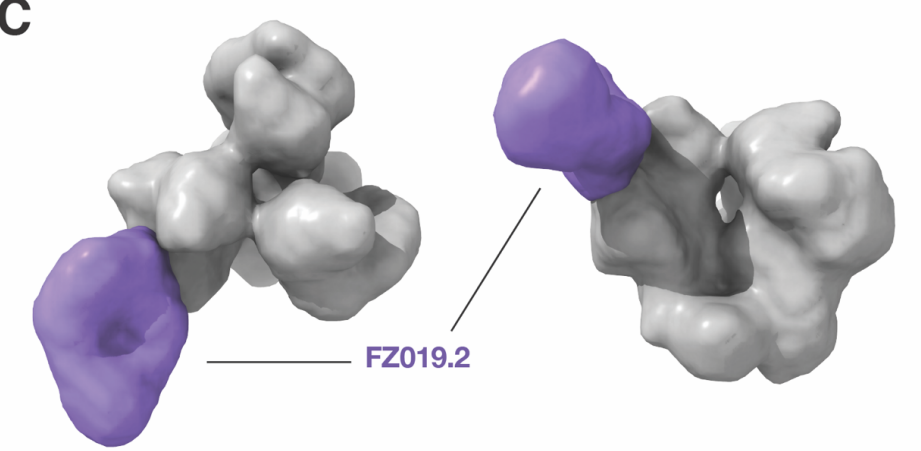

B

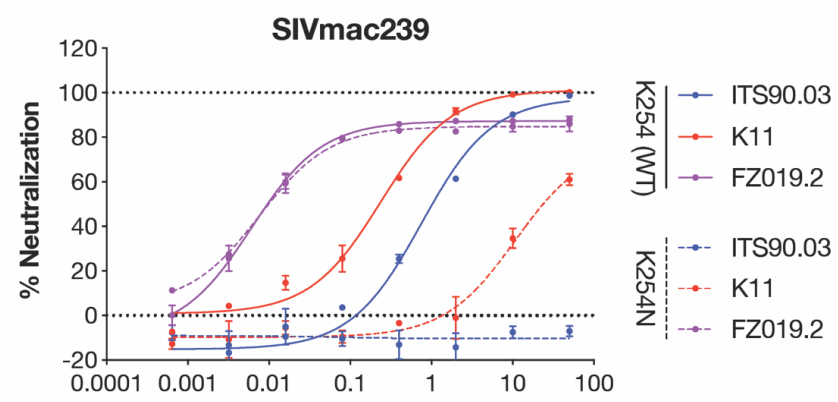

D
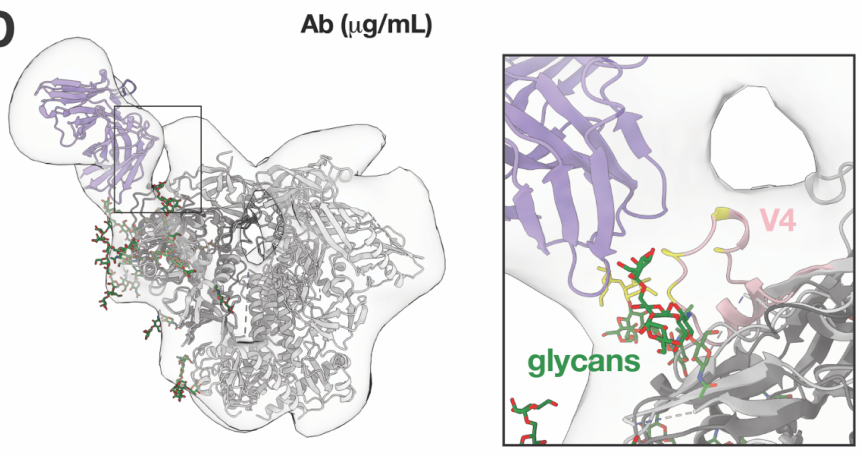

E

\begin{tabular}{|c|c|c|c|c|c|c|c|c|c|c|c|c|c|c|c|c|}
\hline SIV PSV Panel & Tier & Sequence & ITS90.03 & K11 & FZ012.15 & FZ020.1 & FZ020.3 & FZ020.5 & FZ016.3 & FZ016.4 & FZ016.5 & FZ020.7 & FZ020.8 & FZ019.2 & J9 & CD4-IgG2 \\
\hline SIVmac239 & Tier 3 & K254 & 1.176 & 0.240 & 0.345 & 0.854 & 0.247 & 1.611 & 3.512 & 0.579 & 0.738 & 1.993 & 1.347 & 0.011 & 0.212 & 0.081 \\
\hline SIVmac239.cs.23 & Tier 3 & К254 & 1.192 & 0.212 & 0.343 & 0.506 & 0.123 & 1.223 & 2.700 & 0.431 & 1.020 & 1.683 & 1.272 & 0.016 & 0.060 & 0.210 \\
\hline SIVmac251.30 & Tier 2 & K254 & 2.008 & 0.803 & 0.979 & 0.834 & 0.234 & 1.057 & 8.882 & 1.382 & 2.551 & 2.811 & 1.852 & $>50$ & $>50$ & 0.212 \\
\hline SIVmac251.6 & Tier 1 & K254 & 0.406 & 0.266 & 0.372 & 0.270 & 0.026 & 0.328 & 2.063 & 0.445 & 0.426 & 1.090 & 0.798 & $>50$ & $>50$ & 0.007 \\
\hline SIVmac251.H9.15 & Tier 1 & K254 & 0.333 & 0.252 & 0.380 & 0.266 & 0.060 & 0.752 & 0.559 & 0.214 & 0.274 & 0.559 & 0.468 & $>50$ & $>50$ & 0.020 \\
\hline RZj5_9Apr09_EnvPL2.1 & Tier 1 & К254 & 1.511 & 0.330 & 0.470 & 0.434 & 0.164 & 0.780 & 5.321 & 0.225 & 0.745 & 1.658 & 1.882 & $>50$ & $>50$ & 0.516 \\
\hline RZu4 16Apro9 EnvPL1.1 & Tier 2 & K254 & 4.511 & 0.699 & 1.935 & 1.897 & 0.647 & 3.985 & $>50$ & 1.171 & 3.281 & 13.510 & 9.821 & $>50$ & $>50$ & 0.350 \\
\hline FJV_15Nov06_EnvPL2.1 & Tier 3 & N254 & $>50$ & $>50$ & $>50$ & $>50$ & $>50$ & $>50$ & $>50$ & $>50$ & $>50$ & $>50$ & $>50$ & $>50$ & $>50$ & 0.096 \\
\hline SIVsmE660.CR54 -2A5 & Tier 2 & N254 & $>50$ & $>50$ & $>50$ & $>50$ & $>50$ & $>50$ & $>50$ & $>50$ & $>50$ & $>50$ & $>50$ & $>50$ & $>50$ & 0.003 \\
\hline SIVmac251.cs.41 & Tier 2 & N254 & $>50$ & 7.204 & $>50$ & $>50$ & 15.200 & $>50$ & $>50$ & $>50$ & $>50$ & $>50$ & $>50$ & $>50$ & $>50$ & 0.390 \\
\hline RS08_17Jan06 EnvPL1.1 & Tier 2 & N254 & $>50$ & $>50$ & $>50$ & $>50$ & $>50$ & $>50$ & $>50$ & $>50$ & $>50$ & $>50$ & $>50$ & $>50$ & $>50$ & 0.011 \\
\hline FWK 12Aug04_EnvPL4.1 & Tier 2 & N254 & $>50$ & $>50$ & $>50$ & $>50$ & $>50$ & $>50$ & $>50$ & $>50$ & $>50$ & $>50$ & $>50$ & $>50$ & $>50$ & 0.023 \\
\hline SIVsmE660.C3PC -AB & Tier 1 & N254 & $>50$ & $>50$ & $>50$ & $>50$ & $>50$ & $>50$ & $>50$ & $>50$ & $>50$ & $>50$ & $>50$ & $>50$ & $>50$ & 0.002 \\
\hline FFV_18Nov04_EnvPL2.1 & Tier 1 & N254 & $>50$ & $>50$ & $>50$ & $>50$ & $>50$ & $>50$ & $>50$ & $>50$ & $>50$ & $>50$ & $>50$ & $>50$ & $>50$ & 0.052 \\
\hline SIVsmE660.11 & Tier 1 & N254 & 2.056 & $>50$ & $>50$ & $>50$ & $>50$ & $>50$ & $>50$ & $>50$ & $>50$ & $>50$ & $>50$ & $>50$ & $>50$ & 0.007 \\
\hline
\end{tabular}

Figure 2. SIVmac239 nAbs recognize two specificities: a glycan hole and the V4 loop. (A) Negative stain electron microscopy (nsEM) of K11 (red) bound to SIVmac239 SOSIP.664 (gray). (B) Representative neutralization curves of K11 (red), ITS90.03 (blue), and FZ019.2 (purple) against wildtype SIVmac239 virus (solid line) and K254N mutant with N-linked glycan filled in (dashed lines). Each sample is tested in duplicate and error bars represent the standard deviation. (C) nsEM reconstruction of FZ019.2 Fab (purple) bound to SIVmac239 SOSIP.664. (D) Model of the complex using BG505 SOSIP.664 (PDB:6X9R) with the SIVmac239 delta V1/N2 gp120 monomer crystal structure (PDB:6TYB) aligned to one protomer showing that FZ019.2 targets the V4 loop (pink). Residues within $5 \AA$ of the Fab are colored yellow. (E) Neutralization breath and potency $(\mu \mathrm{g} / \mathrm{mL})$ of isolated mAbs against an SIV pseudovirus panel. CD4-lgG2 served as positive control. 


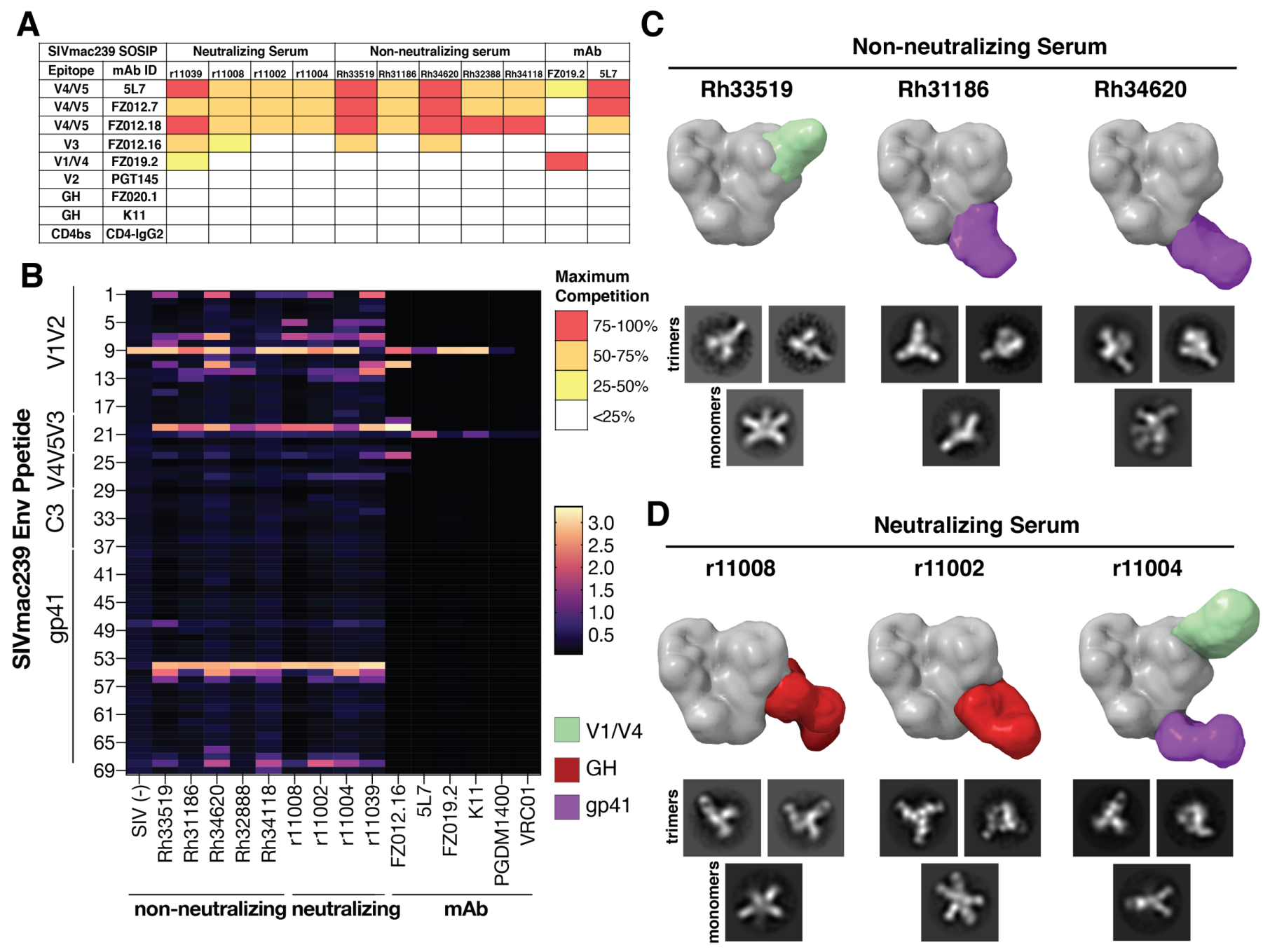

Figure 3. Mapping of polyclonal antibody epitope specificities. (A) ELISA competition between a panel of SIVmac239 Env-binding mAbs and infected sera for binding to SIVmac239 SOSIP. Maximum percentage of competition is colored according to the key. (B) ELISA screening of infected rhesus sera to SIVmac239 Env 15-mer peptides. SIVmac239 neutralizing mAbs (K11, FZ019.2), non-neutralizing mAbs (FZ012.16, 5L7) and HIV bnAbs (PGDM1400, VRC01) served as controls. Absorbance intensity at OD405 nm were colored according to the key. (C-D) EMPEM mapping of rhesus (C) non-neutralizing sera Rh33519, Rh31186, Rh34620 and (D) neutralizing sera r11008, r11002, r11004 for binding to SIVmac239 SOSIP. Shown are representative 2D class averages of trimeric and monomeric particles bound to polyclonal Fabs along with segmentations of 3D reconstructions showing the location of bound Fabs relative to a molecular surface model of the SIVmac239 structure. Epitope specificities are colored according to the key. 

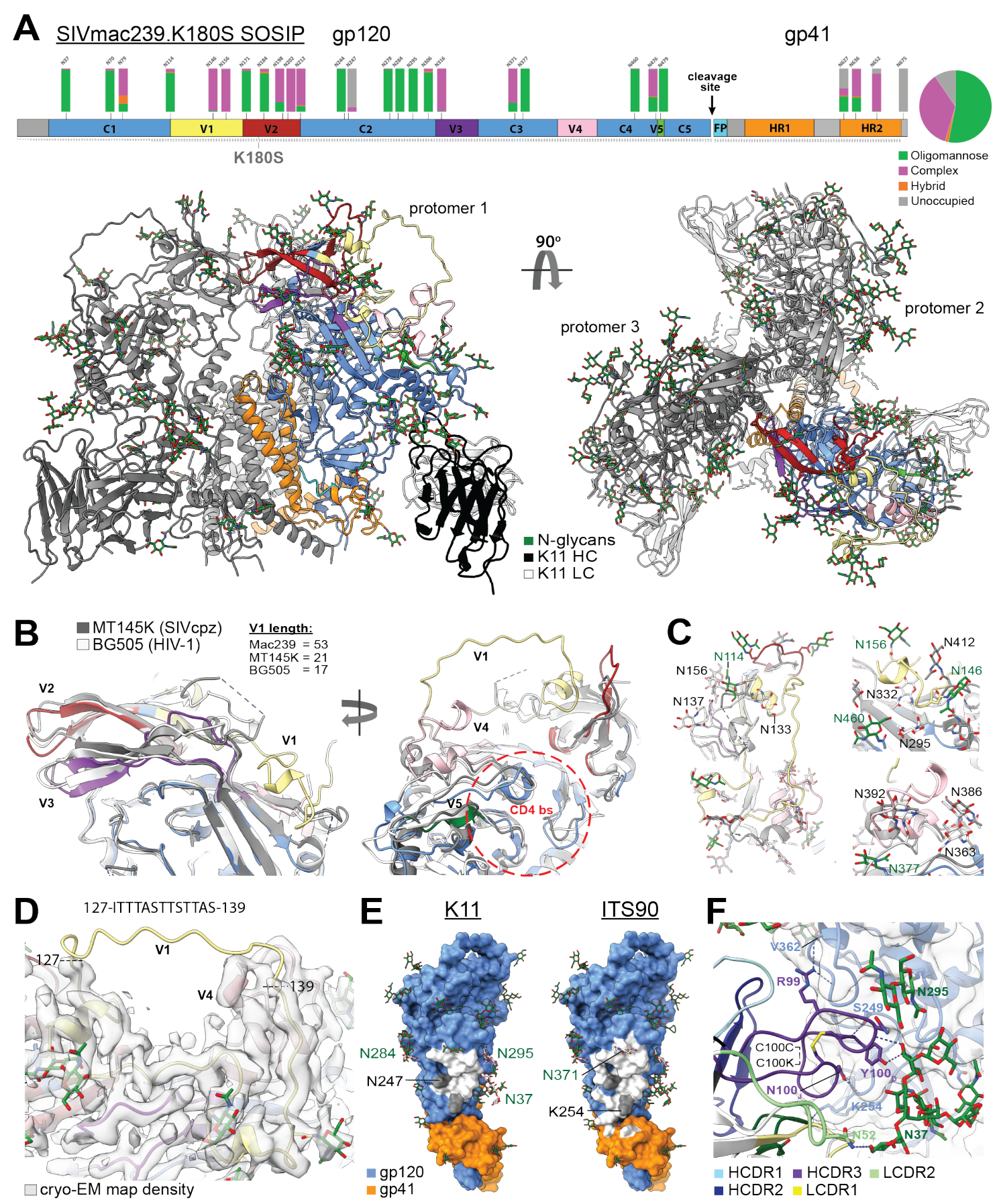

Figure 4. Cryo-EM structure of SIVmac239.K180S SOSIP trimer in complex with the nAb K11. (A) Domain organization, site-specific N-linked glycan distribution, and cryo-EM structure of SIVmac239.K180S SOSIP in complex with K11. (B) Close-up of the V1-V5 loops with the structures of SIVcpz MT145K (PDB:6OHY) and HIV-1 BG505 SOSIP (PDB:6X9R) Env trimers overlayed for comparison - glycans not shown. (C) Close-up views of the novel SIVmac239 V1 and V4 loop conformations showing the positions of N-linked glycans (Asn-NAG only) for all three structures to emphasize the clashes that would occur with HIV and SIVcpz glycans. (D) Cryo-EM map density for the novel V1 and V4 loop conformations with the sequence of the disordered V1 fragment indicated above. (E) Epitopes of both K11 and ITS90.03 (PDB:6TYB) mapped to the SIVmac239 trimer structure (white indicates residues within $5 \AA$ of Fab residues). (F) Close-up view of the K11 epitope-paratope showing hydrogen bonds with gp120 residues and $\mathrm{N}$-linked glycans. Also indicated is the intra-CDRH3 disulfide bond. 
A

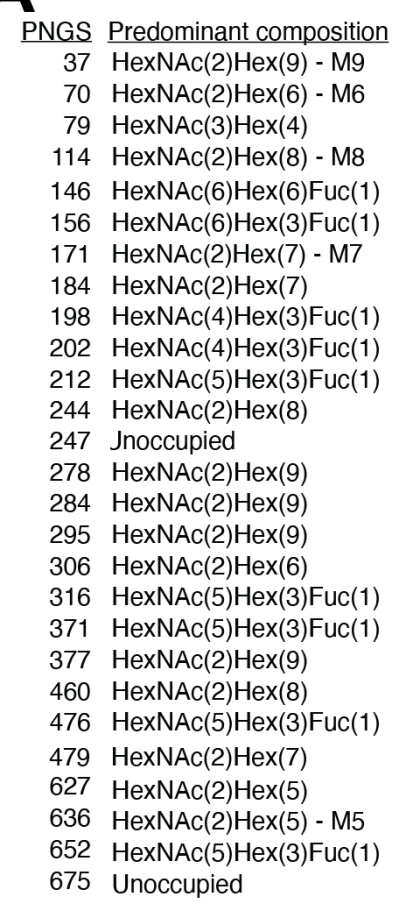

B


complex-type glycans
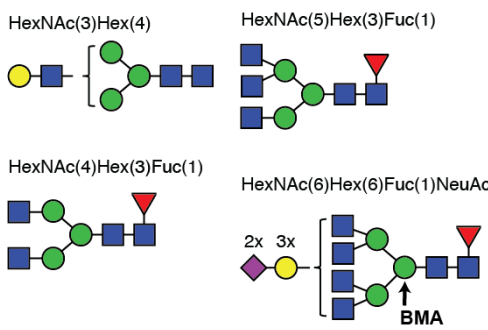

ten fully glycosylated models

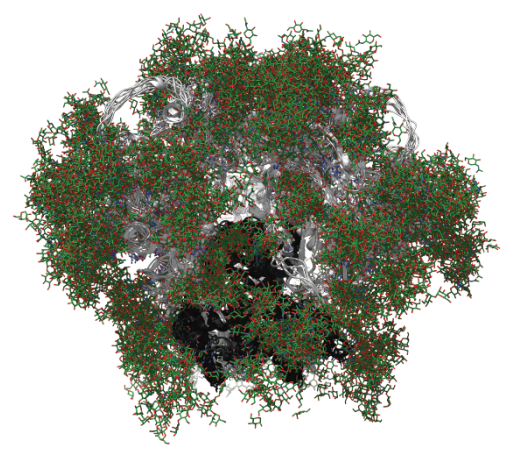

C.
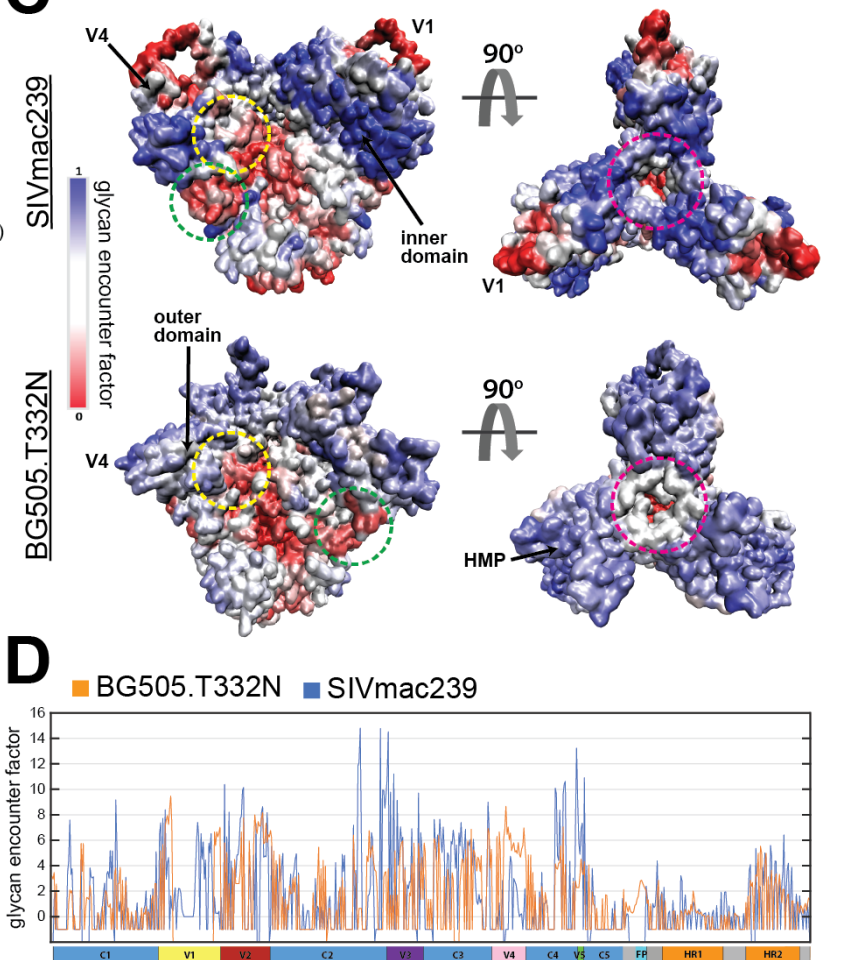

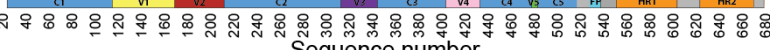
Sequence number

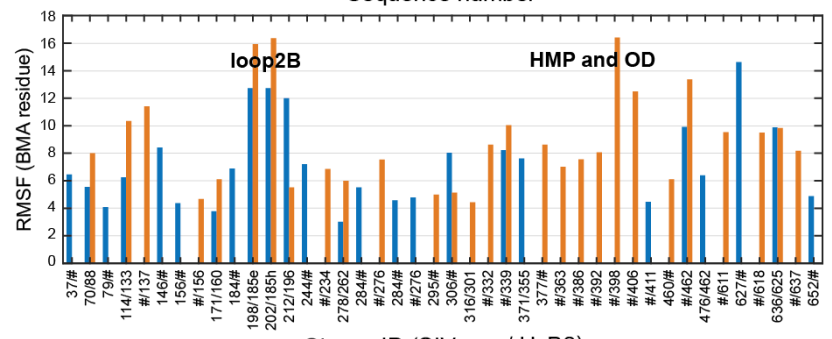

Glycan ID (SIVmac / HxB2)

Figure 5. Site-specific MS and computational modeling of the SIVmac239 Env trimer glycan shield. (A) Predominant glycoforms at each site as determined by MS used for computational modeling along with cartoon representations of each type of complex glycan and a visualization of 10 (1000 total) different fully glycosylated models with glycans in green and gp120 and gp41 in white and black respectively. (B) Glycoform distribution for the two highly under-processed glycan sites at N37 and N278 showing the detection of glucosylated Man9 at both sites along with views of each glycan structure showing stabilizing hydrogen bonds with neighboring protein and glycan residues. (C) Glycan encounter factor calculated for every surface-exposed residue using 1000 fully glycosylated models of both SIVmac239 and BG505 SOSIP.664 and projected as a color map onto the molecular surface representation. Blue indicates the most shielded surfaces and red the most exposed. Demarcated with dashed circles are some prominent regions such as common neutralizing epitopes and the CD4 binding site. (D) Per-residue glycan encounter factor and per-glycan root mean squared fluctuation (RMSF) calculated from 1000 fully glycosylated trimer models. 

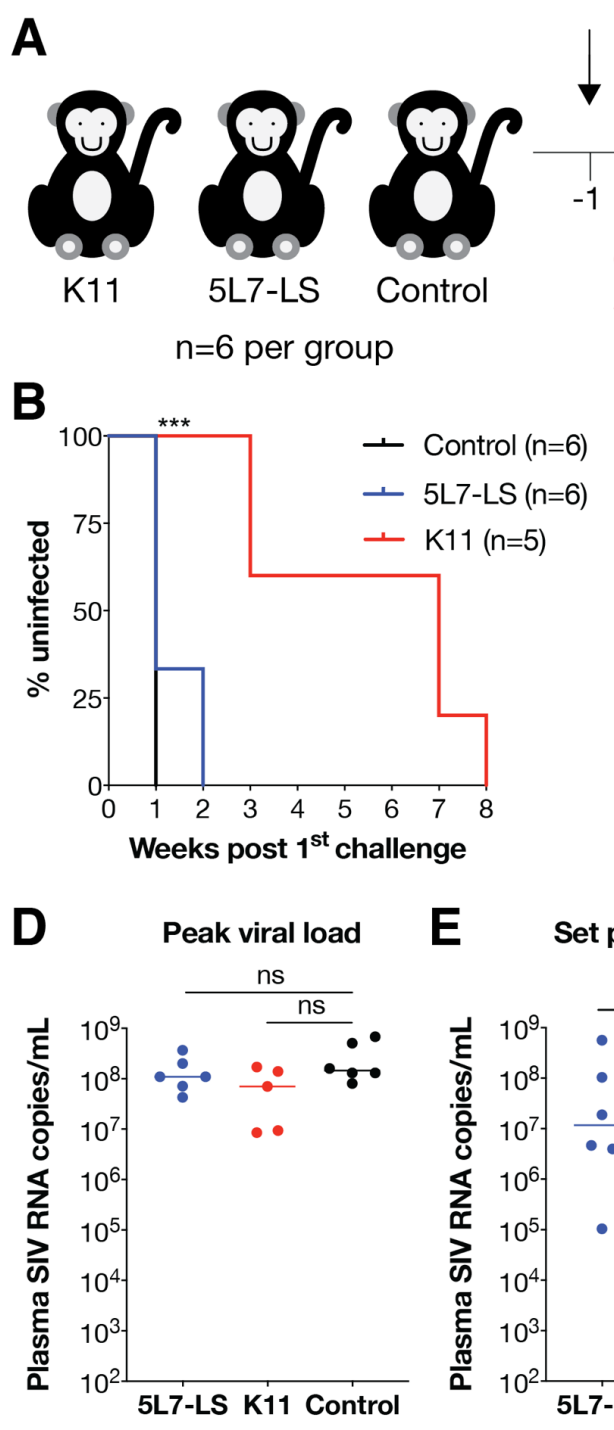



Ab infusion $60 \mathrm{mg} / \mathrm{kg}$
C

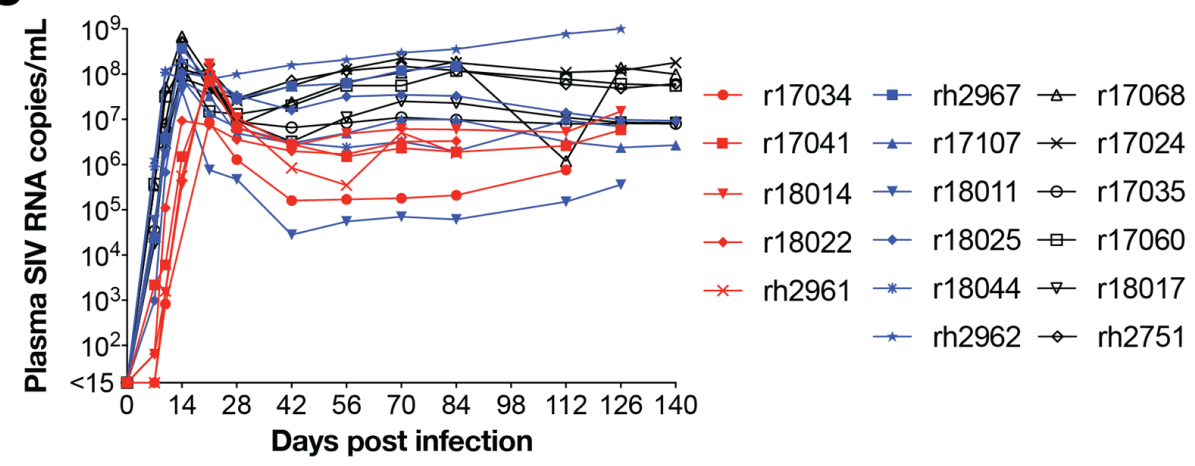

Figure 6. Repeated SIVmac239 IV challenge of 5L7-LS-infused, K11-infused and control animals. (A) SIVmac239 passive Ab protection experimental design. Rhesus macaques were allocated to 3 groups; Group 1 and Group 2 were infused with $60 \mathrm{mg} / \mathrm{kg}$ of 5L7-LS and K11 antibody, respectively, one day prior to challenge. Group 3 animals were untreated controls. Animals were challenged weekly by the intravenous route with a repeat $10^{5.5}$ animal $I_{50}$ of SIVmac239. Blood was drawn and plasma viral load was measured at day 7 and day 10 following each challenge. Animals with detectable viral loads at either of those time points were no longer challenged. After three repeated challenges, a second infusion was given to all Group 1 and Group 2 animals. Uninfected animals were repeatedly challenged intravenously. (B) Kaplan-Meier curves comparing three test groups colored according to the key. Protection was measured by log-rank (Mantel-Cox) test. ${ }^{* * *}: \mathrm{P}<0.001$. ns: not significant. (C) Plasma viral loads after infectious exposure with SIVmac239 in the 3 animal groups: K11 (red), 5L7-LS (blue), and control (black). The threshold of the assay employed was 15 SIV RNA copies/mL. The plasma viral concentrations for each animal were indicated by different symbols. (D) Comparison of peak plasma viral loads among the 3 groups of infected animals based on Mann-Whitney $\mathrm{U}$ test. ns: not significant. (E) Comparison of set point viral load among 3 groups of infected animals based on Mann-Whitney $U$ test. Set point viral load was geometric mean of all values from each animal between weeks 10 and 20. ${ }^{*}: \mathrm{P}<0.01$. ns: not significant. (F) Plasma nAb ID 50 titers and geometric mean of ID 50 titer 7 days prior to infection in the K11 group using the SIVmac239 pseudovirus assay. N/A: not applicable. (G) ADCC activity was measured for rhesus mAbs using SIVmac239 virus on CEM.CCR5 target cells and macaque NK effector cells. CD4-IgG2 served as positive control and an anti-dengue mAb Den3 as a negative control. 


\section{MATERIALS AND METHODS}

\section{Rhesus macaques}

The 18 Indian rhesus macaques (Macaca mulatta) used in the current study (11 males and 7 females) were housed at the Wisconsin National Primate Research Center (WNPRC), cared for in accordance with Weatherall report guidelines, and the principles described in the National Research Council Guide for the Care and Use of Laboratory Animals. All procedures were previously approved by the University of Wisconsin Graduate School Animal Care and Use Committee (animal welfare assurance number 16-00239 [A3368-01]; protocol number G005248). Rhesus macaques were separated as follows: Group 1 (5L7-LS-infused RMs, $n=6$ ), Group 2 (K11-infused RMs, $n=6$ ), Group 3 (untreated RMs, $n=6$ ). Both $5 \mathrm{L7}$-LS and $\mathrm{K} 11$ were prepared in saline bags and administered intravenously into each animal at $60 \mathrm{mg} / \mathrm{kg}$ before the first challenge. Three weeks after, a second infusion was given to all Group 1 and Group 2 animals.

\section{Cell lines}

Irradiated 3T3msCD40L cells were used in single B cell culture assay. TZM-bl cells (NIH AIDS Reagents Program) were used in pseudovirus neutralization assay. Human HEK 293T cells (ATCC) were used for pseudovirus production. HEK 2935 cells (ATCC) were used for the generation of pseudovirus expressing Man5 glycans. FreeStyle HEK 293 cells (ThermoFisher) were used for recombinant Env protein production. Expi293F cells ((ThermoFisher) were used for monoclonal antibody production. CEM.NKR-CCR5-sLTRLuc cells (Alpert et al., 2012) were used for ADCC assay.

\section{Recombinant Env protein purification}

SIVmac239 SOSIP.664-His, SIVmac239 gp140 FT, SIVmac239 gp120 and other Env mutant constructs were transiently expressed in FreeStyle 293F cells (ThermoFisher) as described previously (von Bredow et al., 2019; Gorman et al., 2019). SIVmac239 SOSIP.664 supernatants were purified by affinity chromatography using a PGT145 column. Supernatants of SIVmac239 gp140 FT, SIVmac239 gp120 and other mutant constructs were purified by an agarose bound Galanthus Nivalis Lectin (GNL) column (VectorLab). Affinity-purified proteins were then purified by size exclusion chromatography using a HiLoad 26/600 Superdex 200 pg column (GE Healthcare). 


\section{Isolation of SIVmac239 SOSIP.664-specific memory B cells by flow cytometry}

Cryopreserved PBMCs were thawed, washed, and stained with an antibody cocktail of CD3 (clone SP34-2, BD Biosciences), CD4 (clone OKT4, Biolegend), CD8 (clone RPAT8, BD Biosciences), CD14 (clone M5E2, BD Biosciences), CD20 (clone 2H7, Biolegend), IgM (MHM-88, Biolegend), IgG (clone G18-145, BD Biosciences) and fluorescently labeled biotinylated SIVmac239 SOSIP.664 Env at room temperature for $20 \mathrm{~min}$ in the dark. SIVmac239 Env trimer probes were labeled with two different fluorophores, from which SIVmac239 Env dual positive memory B cells (CD3-CD4-CD8-CD14-CD20+IgMIgG+SIVmac239 SOSIP2+) were analyzed with BD FACSMelody or BD FACSFusion, and single-cell sorted, cultured, expanded in 384-well plates as described previously(Huang et al., 2013; Zhao et al., 2020). Briefly, sorted B cells were cultured with Iscove's modified Dulbecco's medium (IMDM) with GlutaMAX (Gibco) supplemented with 10\% heatinactivated fetal bovine serum (FBS), 1X MycoZap Plus-PR (Lonza), $100 \mathrm{U} / \mathrm{mL}$ human IL-2 (Roche), $50 \mathrm{ng} / \mathrm{mL}$ human IL-21 (Invitrogen), $50 \mathrm{ng} / \mathrm{mL}$ human IL-4 (Miltenyi), 0.1 $\mu \mathrm{g} / \mathrm{mL}$ anti-rhesus $\lg \mathrm{G}(\mathrm{H}+\mathrm{L})$ (BioRad), and irradiated $3 \mathrm{~T} 3 \mathrm{msCD} 40 \mathrm{~L}$ feeder cells. Flow cytometric data were subsequently analyzed using FlowJo (v10.7.1).

\section{SIV pseudovirus production}

The panel of SIV pseudovirus constructs were kind gifts from Mario Roederer at $\mathrm{NIH}$, David Montefiori at Duke University, and Cynthia Derdeyn at Emory University. SIV pseudovirus Env construct was cotransfected with env-deficient backbone plasmid (pSG3 $\triangle E n v$ ) in a 1:2 ratio with transfection reagent FuGENE 6 (Promega) in HEK 293T cells according to manufacturer's instructions. To investigate the role of glycosylation for FZ019.2 antibody neutralization, two approaches were used: glycosidase inhibitors, and the mutant GnT1-deficient HEK $293 \mathrm{~S}$ cell line (GnT1- ) (Doores and Burton, 2010). Glycosidase inhibitors were added at the time of transfection and were used at following concentrations: $25 \mu \mathrm{M}$ kifunensine and $20 \mu \mathrm{M}$ swainsonine. Mutant virus Env plasmid was generated by Quikchange site-directed mutagenesis kit (Agilent) following manufacturer's instructions. After $72 \mathrm{~h}$ of transfection, supernatants containing viruses were harvested and sterile-filtered $(0.22 \mu \mathrm{m})\left(\right.$ EMD Millipore) and frozen at $-80^{\circ} \mathrm{C}$ for long term storage.

\section{Neutralization screening}


After 2 weeks of culture, supernatants were carefully collected and screened in SIVmac239 pseudovirus micro-neutralization assay. $20 \mu \mathrm{L}$ of culture supernatants were incubated with SIVmac239 pseudovirus in sterile 384-well white plates (Greiner Bio-One) for $1 \mathrm{~h}$ at $37^{\circ} \mathrm{C}$. Then $20 \mu \mathrm{L}$ of TZM-bl cells with diluted DEAE-dextran were added to each well at a concentration of 0.2 million cells $/ \mathrm{mL}$ and incubated for $48 \mathrm{~h}$ at $37^{\circ} \mathrm{C}$. After that, culture media were removed, cells were lysed, luciferase activity was measured by adding BrightGlo (Promega) according to manufacturer's instructions. Sorted wells with detectable IgG secretion and over $40-50 \%$ neutralization activity were selected to amplify antibody genes.

\section{RT-PCR, Ig amplification, cloning and antibody production}

After removing culture supernatants, cells in 384-well plates were lysed, and mRNA were extracted using TurboCapture 384 mRNA kit (QIAGEN, 72271) following manufacturer's instructions. mRNA plates were stored at $-80{ }^{\circ} \mathrm{C}$. Single cell RNA from neutralization positive wells were reverse transcribed as described previously (Wu et al., 2010). Subsequently, nested PCR reactions of Ig heavy chain and light chain variable regions were performed in $25 \mu \mathrm{L}$ volume with $2 \mu \mathrm{L}$ cDNA transcript. For the first round of PCR in $25 \mu \mathrm{L}$ reaction, $2.5 \mu \mathrm{L}$ of $10 X$ PCR buffer (QIAGEN), $0.25 \mu \mathrm{L}$ of HotStarTaq Plus DNA Polymerase (QIAGEN), $0.5 \mu \mathrm{L}$ of dNTP (10 mM) (ThermoFisher), $0.25 \mu \mathrm{L}$ of $\mathrm{MgCl} 2$ $(25 \mu \mathrm{M}), 0.25 \mu \mathrm{L}$ of forward primer mixture (50 $\mu \mathrm{M}$ each primer), $0.25 \mu \mathrm{L}$ of reverse primer mixture (25 $\mu \mathrm{M}$ each primer), $19 \mu \mathrm{L}$ of DEPC-treated water (ThermoFisher), $2 \mu \mathrm{L}$ of gene transcripts. Rhesus primers and PCR program were described previously (Gorman et al., 2019). $2 \mu \mathrm{L}$ of PCR1 products were amplified by nested PCR using $5 \mu \mathrm{L}$ of 5X Phusion HF buffer (Thermo Fisher), $0.24 \mu \mathrm{L}$ of Phusion HF DNA polymerase ( $2 \mathrm{U} / \mu \mathrm{L}$, Thermo Fisher), $0.5 \mu \mathrm{L}$ of $\mathrm{dNTP}, 0.25 \mu \mathrm{L}$ of forward primers and $0.25 \mu \mathrm{L}$ of reverse primers with Gibson adaptor sequences, $0.75 \mu \mathrm{L}$ of $2 \mathrm{X} \mathrm{MgCl}_{2}$, using the following PCR program: $30 \mathrm{~s}$ at $98^{\circ} \mathrm{C}$, followed by 35 cycles of $10 \mathrm{~s}$ at $98^{\circ} \mathrm{C}, 40 \mathrm{~s}$ at $72^{\circ} \mathrm{C}$, and a final extension for 5 min. Amplified PCR products were analyzed with $2 \% 96 \mathrm{E}$-gel (ThermoFisher). Antibodies with recovered and paired heavy chains and light chains were cloned into the corresponding $\lg \gamma 1$, $\lg \kappa, \lg \lambda$ expression vectors (Wu et al., 2010). Cloned rhesus heavy chain and light chain variable regions were sequenced (Genewiz) and subsequently analyzed using the rhesus germline database (Cirelli et al., 2019). 
Antibody heavy chain and light chain constructs were transiently expressed with the Expi293 Expression System (ThermoFisher). HC and LC plasmids were cotransfected at a 1:2.5 ratio with transfection reagent FectoPRO (Polyplus) in Expi293 cells according to the manufacturer's instructions. $24 \mathrm{~h}$ post transfection, cells were fed with $300 \mathrm{mM}$ valproic acid and $40 \%$ glucose (Gibco). After 4 to 5 days of transfection, cell supernatants were harvested and sterile filtered $(0.22 \mu \mathrm{m})$. Antibody was purified by Protein A Sepharose (GE Healthcare) as described previously (Sok et al., 2013).

\section{TZM-bl neutralization assay}

Serially diluted serum or antibody sample was incubated with SIV pseudovirus in halfarea 96-well white plates using Dulbecco's Modified Eagle Medium (IMDM) (Gibco) supplemented with 10\% FBS, $2 \mathrm{mM}$ L-glutamine (Gibco), $100 \mathrm{U} / \mathrm{mL}$ Penicillin/Streptomycin (Gibco). After $1 \mathrm{~h}$ incubation at $37^{\circ} \mathrm{C}$, TZM-bl cells with diluted DEAE-dextran were added onto the plates at 200,000 cells/well. For testing antibody neutralization against replication competent virus, serially diluted $m A b s$ were incubated with replication competent SIVmac239 for $1 \mathrm{~h}$ at at $37^{\circ} \mathrm{C}$, then transferred onto TZM-bl cells containing $1 \mu \mathrm{M}$ Indinavir (Sigma) in half-area 96-well plates (Corning). After $48 \mathrm{~h}$ of incubation, culture supernatants were removed, cells were lysed in luciferase lysis buffer (25 mM Gly-Gly pH 7.8, 15 mM MgSO 4 , 4 MM EGTA, 1\% Triton X-100). Luciferase activity was measured by adding BrightGlo (Promega) according to the manufacturer's instructions. Assays were tested in duplicate wells and independently repeated at least twice. Neutralization IC50 or ID50 titers were calculated using "One-Site Fit LogIC I0" $_{5}$ regression in GraphPad Prism 8.0. Maximum Percentage of Neutralization (MPN) was defined as the maximum \% neutralization observed at the highest mAb concentrations tested.

\section{Recombinant protein binding ELISA}

6x His tag monoclonal antibody (Invitrogen) or streptavidin (Jackson ImmunoResearch) was coated at $2 \mu \mathrm{g} / \mathrm{mL}$ in PBS onto half-area 96-well high binding plates (Corning) overnight at $4^{\circ} \mathrm{C}$. After washing, plates were blocked with PBS/3\% BSA for $1 \mathrm{~h}$ at RT. Histagged recombinant SIVmac239 Env protein was added at $1 \mu \mathrm{g} / \mathrm{mL}$ onto plates precoated with 6x His tag antibody while biotinylated SIVmac239 Env protein was added at $1 \mu \mathrm{g} / \mathrm{mL}$ onto plates precoated with streptavidin. Protein was captured and incubated for $1 \mathrm{~h}$ at RT. After washing, serially diluted mAb in PBS/1\%BSA was added in duplicates into wells and 
incubated for $1 \mathrm{~h}$ at RT. After washing, 1:1000 diluted alkaline phosphatase-conjugated goat anti-human IgG Fc $\gamma$ antibody (Jackson ImmunoResearch) was added into wells and incubated at RT for $1 \mathrm{~h}$. After final wash, phosphatase substrate (Sigma-Aldrich) was added into wells. Absorption was measured at $405 \mathrm{~nm}$. Non-linear regression curves were analyzed using Prism 8 software to calculate $\mathrm{EC}_{50}$ values.

\section{Competition ELISA}

6x His tag monoclonal antibody (Invitrogen) was coated at $2 \mu \mathrm{g} / \mathrm{mL}$ in PBS onto half-area 96-well high binding plates (Corning) overnight at $4^{\circ} \mathrm{C}$. After washing, plates were blocked with PBS/3\% BSA for at least $1 \mathrm{~h}$ at RT. His-tagged SIVmac239 SOSIP.664 protein was added into wells at $1 \mu \mathrm{g} / \mathrm{mL}$ and incubated for $1 \mathrm{~h}$ at RT. After washing, serially diluted $\mathrm{mAbs}$ (started at $50 \mu \mathrm{g} / \mathrm{mL}$ ) or sera (started at 1:50 dilution) in PBS/1\% BSA were added into wells and incubated for $30 \mathrm{~min}$ at RT. After removing liquid, biotinylated mAb was added at a concentration corresponding to $\mathrm{EC}_{70}$ and incubated for $45-60 \mathrm{~min}$. After washing, alkaline phosphatase-conjugated streptavidin (Jackson ImmunoResearch) was diluted at $1: 1000$ in PBS/1\% BSA and added into the wells at RT for $1 \mathrm{~h}$. After the final wash, phosphatase substrate (Sigma-Aldrich) was added into the wells. Absorption was measured at $405 \mathrm{~nm}$.

\section{Negative stain Electron Microscopy (nsEM) sample preparation, imaging, and data processing.}

Monoclonal antibody was digested into Fab using papain resin and purified by protein $\mathrm{A}$ beads (ThermoFisher) according to the manufacturer's instructions. Rhesus sera or plasma antibodies were first purified by affinity chromatography using Protein A Sepharose (GE Healthcare) according to the manufacturer's instructions. Then polyclonal serum antibodies were digested into Fab using papain resin. Purified SIVmac239 trimer was then mixed with purified Fab or IgG and incubated overnight followed by purification with size exclusion chromatography. Antibodies were added at a $6 x$ molar excess to trimer for monoclonal complexes and $\sim 30 x$ molar excess for polyclonal complexes. Purified complexes were then concentrated to $\sim 0.01 \mathrm{mg} / \mathrm{mL}$ and applied to plasma cleaned carbon grids followed by two rounds of staining with uranyl formate (UF). Imaging was performed on an FEI Tecnai Spirit T12 transmission electron microscope operating at $120 \mathrm{keV}$ and equipped with an Eagle 4K CCD. A magnification of 52,000x was used, resulting in a physical pixel size of $2.05 \AA$. Automated data collection was performed using 
the Leginon software package (Suloway et al., 2005). All single-particle data processing steps were performed using Relion/3.0 (Zivanov et al., 2018). Figures were generated using UCSF Chimera (Pettersen et al., 2004) by aligning representative 3D reconstructions to a ligand-free molecular surface model of SIVmac239, followed by segmentation of Fab regions using Segger (Pintilie and Chiu, 2012). For clarity, figures only display one Fab density per epitope.

\section{Cryo-EM sample preparation and imaging.}

Purified K11 lgG was mixed with purified SIVmav239 trimer then complexes were immediately purified via SEC and concentrated to $\sim 1 \mathrm{mg} / \mathrm{mL}$ for cryo-EM preparation. Concentrated sample was mixed with $0.5 \mu \mathrm{L}$ of $0.04 \mathrm{mM}$ lauryl maltose neopentyl glycol (LMNG; Anatrace) to a final concentration of $0.005 \mathrm{mM}$ and $4 \mu \mathrm{L}$ of this solution was applied to plasma-cleaned 1.2/1.3 C-Flat holey carbon grids (Electron Microscopy Sciences) using a Vitrobot mark IV (Thermo Fisher Scientific) with a $7 \mathrm{sec}$ blot time, 0 blot force, and wait time of 0 sec. Prepared grids were then stored in liquid nitrogen until they were transferred to a microscope for imaging. A table of detailed imaging conditions is presented in Table S2. Data was collected with Leginon automated microscopy software (Suloway et al., 2005) on an FEl Titan Krios operating at $300 \mathrm{keV}$ (Thermo Fisher Scientific) equipped with a K2 Summit direct electron detector (Gatan) operated in counting mode.

\section{Cryo-EM data processing.}

All movie micrographs were aligned and dose-weighted using MotionCor2 (Zheng et al., 2017) and CTF parameters were estimated with GCTF (Zhang, 2016). Single-particle processing was carried out using CryoSparc2 (Punjani et al., 2017). The following general workflow was used for single-particle data processing. After frame alignment, doseweighting, and CTF estimation, micrographs were sorted based on CTF fit parameters and particle picking was performed first using a gaussian blot template on a subset of micrographs. These particles were then extracted, aligned and classified in 2-D, and the class averages were then used for template picking of the full dataset. Picked particles were extracted and subjected to one or two rounds of 2D-classificaiton followed by subset selection and re-extraction with re-centering. One round of ab initio classification was carried out followed by subset selection of all classes containing well-refined trimers. After subset selection, 3D-autorefinement was performed with per-particle CTF estimation 
using C3 symmetry. It was observed that the map density for gp41 was very poorly resolved so we performed another round of 3-D classification, this time using the newly developed 3-D variability algorithm implemented in CryoSparc2 (Punjani and Fleet, 2021) followed by clustering into 6 classes. Analysis of the variability along principal components 1-3 revealed a significant "scissoring" motion (Supplemental Movie 1) arising from movements in the HR1 and HR2 alpha helices. Clusters with clear well resolved gp41 helices in at least one protomer interface were then pooled and refined again together without imposing any symmetry using non-uniform refinement with per-particle CTF estimation and correction.

\section{Model building and figure preparation.}

Model building was initiated by preparing a monomeric SIVmac239 homology model with SWISS-MODEL (Waterhouse et al., 2018) using MT145K (PDBID:60HY) as a template. A homology model of $\mathrm{K} 11$ was generated using SAbPred (Dunbar et al., 2016). Preliminary Env and Fab models were then fit into the asymmetrically refined cryo-EM map using the protomer with the best resolved gp41 domain and combined into a single PDB file using UCSF Chimera (Pettersen et al., 2004). The novel V1 loop conformation was built by hand along with all glycans using COOT (Emsley and Crispin, 2018), and gp41 helices were adjusted using rigid body docking. Fully glycosylated models were then refined in Rosetta asking for $\sim 300$ models (Wang et al., 2016). All models were validated using MolProbity (Chen et al., 2010) and EMRinger (Barad et al., 2015) and the model with the best combined score was selected. The model was then checked and adjusted manually in COOT and re-refined with Rosetta, if necessary, then renumbered to match Kabat and HXB2 numbering schemes in the python scripting interface of COOT. The protomer with the best resolved gp41 domain from the asymmetric reconstruction was then fit and relaxed into the C3 symmetric map and refined with Rosetta. Final models were then scored again with MolProbity and EMRinger, while glycan structures were further validated with Privateer (Agirre et al., 2015). Figures were prepared with either UCSF Chimera or ChimeraX (Pettersen et al., 2021). Hydrogen bonds were calculated and displayed with UCSF ChimeraX. Volume segmentation was performed with Segger (Pintilie et al., 2010) as implemented in UCSF ChimeraX.

\section{Modeling to quantify the extent of glycan shielding of the SIV mac239 Env surface}


We first generated an ensemble of non-glycosylated protein models using Rosetta multicycle refinement (Herzik et al., 2019; Wang et al., 2016) with our 3.4Å-resolution cryo-EM map and chose the top 10 models based on a combined EMRinger (Barad et al., 2015) and MolProbity (Chen et al., 2010) score to serve as scaffolds. We then built 100 possible glycosylated conformations on to each scaffold, and relaxed them over several steps of conjugate gradient energy minimization and simulated annealing, using our HTAM pipeline(Berndsen et al., 2020). Glycosylation at each PNGS was modeled based on the site-specific predominant glycan type as determined by MS. These 1000 different conformations formed the final glycoprotein ensemble. From this ensemble we calculated the root mean squared fluctuation of each glycan, averaged over the carbon and oxygen atoms of the $\beta$-mannose ring. The per-residue glycan encounter factor (GEF) score (Chakraborty et al., 2020), which quantifies the shielding effect over the protein surface, was calculated as the geometric mean of the probability that surface orthogonal vectors (normal and planar) located at each residue would encounter glycan heavy atoms. For comparison we also performed the same calculations on an equally sized ensemble of fully glycosylated BG505 SOSIP.664 models.

\section{Site-specific glycan analysis}

Three aliquots of SIVmac239 were denatured for $1 \mathrm{~h}$ in $50 \mathrm{mM}$ Tris/HCl, $\mathrm{pH} 8.0$ containing $6 \mathrm{M}$ of urea and $5 \mathrm{mM}$ dithiothreitol (DTT). Next, Env proteins were reduced and alkylated by adding $20 \mathrm{mM}$ iodoacetamide (IAA) and incubated for $1 \mathrm{~h}$ in the dark, followed by a $1 \mathrm{~h}$ incubation with $20 \mathrm{mM}$ DTT to eliminate residual IAA. The alkylated Env proteins were buffer-exchanged into $50 \mathrm{mM}$ Tris/ $\mathrm{HCl}, \mathrm{pH} 8.0$ using Vivaspin columns (3 kDa) and two of the aliquots were digested separately overnight using trypsin, chymotrypsin (Mass Spectrometry Grade, Promega) or alpha lytic protease (Sigma Aldrich) at a ratio of 1:30 $(w / w)$. The next day, the peptides were dried and extracted using C18 Zip-tip (MerckMilipore). The peptides were dried again, re-suspended in $0.1 \%$ formic acid and analyzed by nanoLC-ESI MS with an Ultimate 3000 HPLC (Thermo Fisher Scientific) system coupled to an Orbitrap Eclipse mass spectrometer (Thermo Fisher Scientific) using stepped higher energy collision-induced dissociation (HCD) fragmentation. Peptides were separated using an EasySpray PepMap RSLC C18 column (75 $\mu \mathrm{m} \times 75$ $\mathrm{cm}$ ). A trapping column (PepMap $100 \mathrm{C} 183 \mu \mathrm{M} 75 \mu \mathrm{M} \times 2 \mathrm{~cm}$ ) was used in line with the LC prior to separation with the analytical column. The LC conditions were as follows: 280 minute linear gradient consisting of $4-32 \%$ acetonitrile in $0.1 \%$ formic acid over 260 
minutes followed by 20 minutes of alternating $76 \%$ acetonitrile in $0.1 \%$ formic acid and $4 \%$ Acn in $0.1 \%$ formic acid, used to ensure all the sample had eluted from the column. The flow rate was set to $300 \mathrm{~nL} / \mathrm{min}$. The spray voltage was set to $2.5 \mathrm{kV}$ and the temperature of the heated capillary was set to $40{ }^{\circ} \mathrm{C}$. The ion transfer tube temperature was set to $275^{\circ} \mathrm{C}$. The scan range was $375-1500 \mathrm{~m} / \mathrm{z}$. Stepped HCD collision energy was set to 15 , 25 and $45 \%$ and the MS2 for each energy was combined. Precursor and fragment detection were performed using an Orbitrap at a resolution MS1=120,000. MS2=30,000. The AGC target for MS1 was set to standard and injection time set to auto which involves the system setting the two parameters to maximize sensitivity while maintaining cycle time. Full LC and MS methodology can be extracted from the appropriate Raw file using XCalibur FreeStyle software or upon request.

Glycopeptide fragmentation data were extracted from the raw file using Byos (Version 3.5; Protein Metrics Inc.). The glycopeptide fragmentation data were evaluated manually for each glycopeptide; the peptide was scored as true-positive when the correct $b$ and $y$ fragment ions were observed along with oxonium ions corresponding to the glycan identified. The MS data was searched using the Protein Metrics $305 \mathrm{~N}$-glycan library with sulfated glycans added manually. The relative amounts of each glycan at each site as well as the unoccupied proportion were determined by comparing the extracted chromatographic areas for different glycotypes with an identical peptide sequence. All charge states for a single glycopeptide were summed. The precursor mass tolerance was set at 4 ppm and 10 ppm for fragments. A 1\% false discovery rate (FDR) was applied. The relative amounts of each glycan at each site as well as the unoccupied proportion were determined by comparing the extracted ion chromatographic areas for different glycopeptides with an identical peptide sequence. Glycans were categorized according to the composition detected.

HexNAc(2)Hex(10+) was defined as M9Glc, HexNAc(2)Hex(9-5) was classified as M9 to M3. Any of these structures containing a fucose were categorized as FM (fucosylated mannose). HexNAc(3)Hex(5-6)X was classified as Hybrid with HexNAc(3)Hex(56)Fuc(1)X classified as Fhybrid. Complex-type glycans were classified according to the number of HexNAc subunits and the presence or absence of fucosylation. As this fragmentation method does not provide linkage information compositional isomers are grouped, so for example a triantennary glycan contains HexNAc 5 but so does a 
biantennary glycans with a bisect. Core glycans refer to truncated structures smaller than M3. M9glc- M4 were classified as oligomannose-type glycans. Glycans containing at least one sialic acid or one sulfate group were categorized as NeuAc and sulfated respectively.

\section{SIVmac239 Env peptide ELISA screening}

Biotinylated SIVmac239 Env 15-mer peptides were synthesized at GenScript. Overlapping peptide sequences were shown in Table S3. Streptavidin (Jackson ImmunoResearch) was coated at $2 \mu \mathrm{g} / \mathrm{mL}$ in PBS onto half-area 96-well high binding plates (Corning) overnight at $4^{\circ} \mathrm{C}$. After washing, plates were blocked with $\mathrm{PBS} / 3 \% \mathrm{BSA}$ for $1 \mathrm{~h}$ at RT. After washing, the peptide library was diluted to $50 \mu \mathrm{g} / \mathrm{mL}$ in PBS and added onto individual wells of ELISA plates and incubated for $1 \mathrm{~h}$. After washing, 1:50 diluted rhesus sera or $10 \mu \mathrm{g} / \mathrm{mL}$ mAbs were added to each plate and incubated for $1 \mathrm{~h}$ at RT. After washing, 1:1000 diluted alkaline phosphatase-conjugated goat anti-human IgG Fc $\gamma$ antibody (Jackson ImmunoResearch) was added into wells and incubated at RT for $1 \mathrm{~h}$. After the final wash, phosphatase substrate (Sigma-Aldrich) was added into wells. Absorption was measured at $405 \mathrm{~nm}$.

\section{ADCC assay}

CEM.NKR-CCR5-SLTR-Luc cells (Alpert et al., 2012), which express luciferase (Luc) upon infection, were infected with SIVmac239. After two days of infection, serially diluted mAbs were added to the target cells and incubated for $15 \mathrm{~min}$. Primary human NK cells were added for an approximate 10 to 1 effector to target ratio. Cells were incubated overnight. Luc activity was measured using BrightGlo (Promega) as described above. Uninfected or infected cells incubated with NK cells in the absence of antibody were used to determine background and maximal Luc activity, respectively. The dose-dependent loss of Luc activity represents the antibody-dependent killing of productively infected target cells.

\section{SIVmac239 challenge}

All animals were subjected to repeated intravenous inoculations of $10^{5.5}$ rhesus monkey infectious doses5o per mL of SIVmac239 (Genbank ID M33262). The challenge virus was administered in $1 \mathrm{~mL}$ of PBS. Intravenous challenge occurred weekly. Plasma samples 
were collected at days 7 and 10 post-challenge to assess for viral loads. Animals with detectable viral loads at either of those time points were no longer challenged.

\section{Viral load measurement}

Viral loads were measured using $0.5 \mathrm{~mL}$ of plasma, as previously described (Li et al., 2016). Total RNA was extracted from each sample using QIAgen DSP virus/pathogen Midi kits (QIAGEN) on a QIASymphonyXP laboratory automation instrument platform. A two-step RT PCR reaction was performed using random primed reverse transcription reaction, followed by 45 PCR cycles. Each sample had six replicates. The following primers and probe were used: forward primer: SGAG21: 5'GTCTGCGTCAT(dP)TGGTGCA TTC-3'; reverse primer SGAG22: 5'CACTAG(dK)TGTCTCTGCACTAT(dP)TGTTTTG-3'; probe: PSGAG23: 5'-FAM528 CTTC(dP)TCAGT(dK)TGTTTCACTTTCTCTTCTGCG-BHQ1- 3'. As performed, thethreshold sensitivity of the assay was 15 SIV RNA copies/mL plasma.

\section{Plasma antibody ELISA}

Plasma antibody levels of $\mathrm{K} 11$ and C9-tagged $5 \mathrm{~L} 7$ were measured by ELISA using plates coated overnight at $2 \mu \mathrm{g} / \mathrm{mL}$ with either purified SIVmac239 gp140 FT or mouse antirhodopsin (C9) monoclonal Ab (EMD Millipore, 6B9575), respectively. Following overnight coating, plates were washed with 1 X PBS-Tween20 and blocked with $5 \%$ nonfat dry milk in PBS at $37^{\circ} \mathrm{C}$ for one hour. After blocking, plates were washed and $50 \mu \mathrm{L}$ of serially diluted serum and purified $\mathrm{K} 11$ or $5 \mathrm{~L} 7$ was added to the corresponding wells. Plates were then washed before the adding goat anti-human IgG-HRP (SouthernBiotech, 2045-05) diluted 1:10,000 to all wells and incubating for $1 \mathrm{~h}$ at $37^{\circ} \mathrm{C}$. After a final wash, plates were developed using 3,3',5,5'-Tetramethylbenzidine substrate (SouthernBiotech, 0410-01) and the reaction was stopped using TMB stop solution (SouthernBiotech, 041201 ) before reading absorbance at $450 \mathrm{~nm}$.

\section{STATISTICAL ANALYSIS}

General quantification and standard deviations were calculated between technical replicates using GraphPad Prism 8.0. The Kaplan-Meier method and log-rank test were used to determine whether infusion of K11 affected the acquisition of SIVmac239. The time to detectable infection was analyzed using Kaplan-Meier method, and the differences between K11 group and control group, or between 5L7-LS group and control 
bioRxiv preprint doi: https://doi.org/10.1101/2021.12.22.473937; this version posted December 23, 2021. The copyright holder for this preprint (which was not certified by peer review) is the author/funder. All rights reserved. No reuse allowed without permission.

group were evaluated using log-rank test. The Mann-Whitney test was used to compare the peak viral load of infected animals in K11 or 5L7-LS group with the control group. 
A

\begin{tabular}{|c|c|c|c|c|c|c|}
\hline Rhesus ID & SIVmac239 & SIVmacE543 & $\begin{array}{r}\text { HIV-2 } \\
7312 A\end{array}$ & SIVcpzPtt & BG505 & MLV \\
\hline r11039 & 5,385 & 107,423 & 131,961 & $<50$ & $<50$ & $<50$ \\
\hline r10027 & 169 & 73,260 & 8,945 & $<50$ & $<50$ & $<50$ \\
\hline $\mathrm{r} 11008$ & 1,562 & 21,777 & 52,882 & $<50$ & $<50$ & $<50$ \\
\hline r11002 & 2,061 & 35,398 & 6,653 & $<50$ & $<50$ & $<50$ \\
\hline r04161 & 351 & $>150,000$ & 7,348 & $<50$ & $<50$ & $<50$ \\
\hline r10014 & 213 & 21,372 & 4,819 & $<50$ & $<50$ & $<50$ \\
\hline r03145 & 3,717 & 38,850 & 2,486 & $<50$ & $<50$ & $<50$ \\
\hline r10051 & 3,377 & NT & NT & NT & NT & $<50$ \\
\hline rhBB35 & 676 & NT & NT & NT & NT & $<50$ \\
\hline r10055 & 672 & NT & NT & NT & NT & $<50$ \\
\hline r01007 & 4,101 & NT & NT & NT & NT & NT \\
\hline r09004 & 325 & NT & NT & NT & NT & NT \\
\hline r08037 & 299 & NT & NT & NT & NT & NT \\
\hline
\end{tabular}

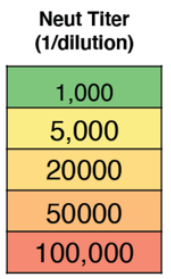

B
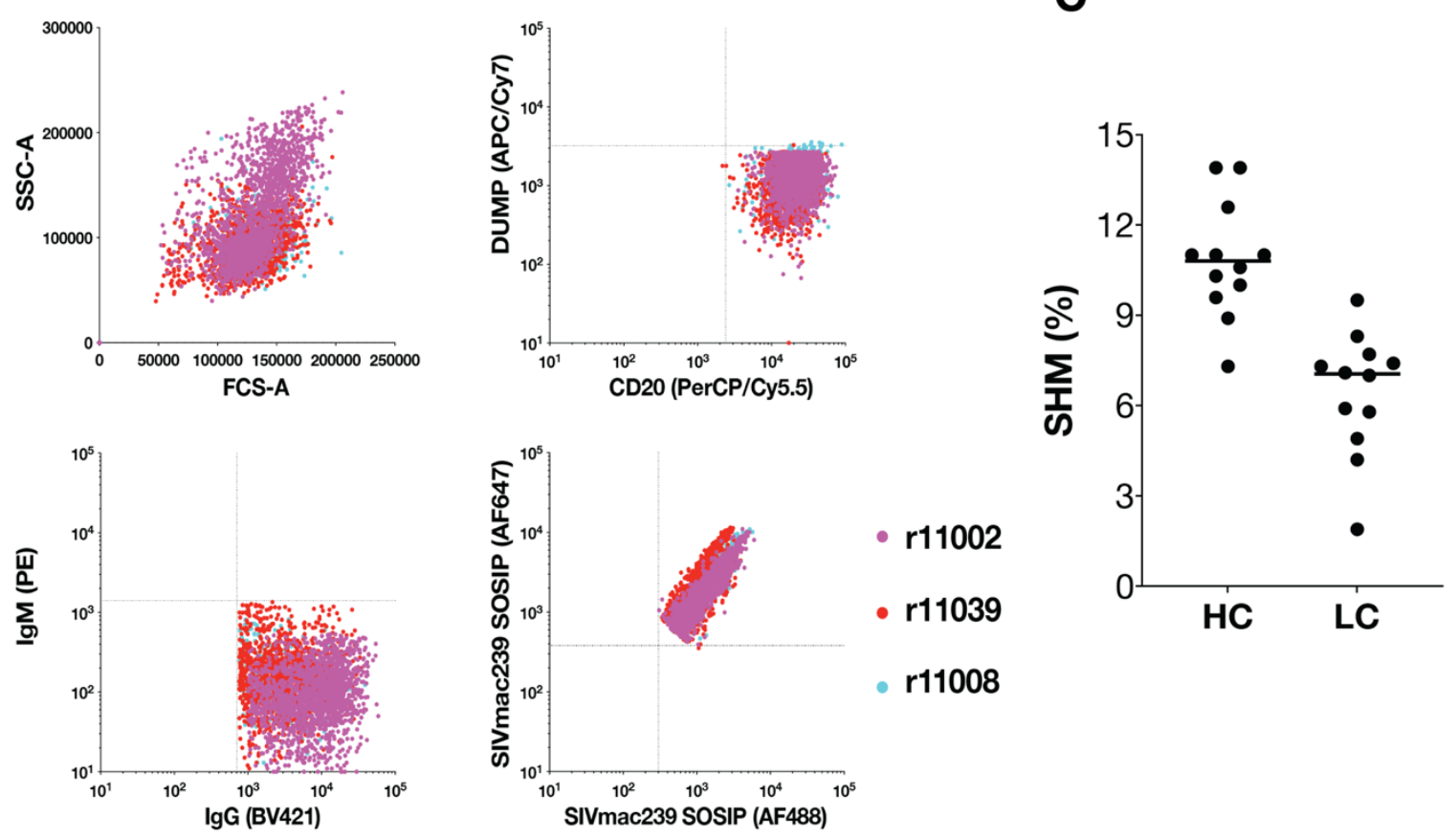

Figure S1. Rhesus plasma neutralization titers and FACS sorting of PBMCs from 3 animals. (A) Neutralization profile of plasma from SIVmac239 infected rhesus macaques against a panel of pseudoviruses including rhesus macaque SIV (SIVmac239, SIVmacE543), an HIV-2 (HIV-2.7312A), a chimpanzee SIV (SIVcpzPtt), an HIV-1 (BG505), and MLV as a control, showing the ID50 of plasma against each virus. N/T: not tested. (B) Index analysis of single-cell sorted CD20+lgM-lgG ${ }^{+}$SIVmac239 SOSIP++ population from the PBMCs of r11002 (pink), r11008 (red), and r11039 (cyan). (C) Somatic hypermutation (SHM) rates of isolated SIVmac239 mAbs at the nucleotide level. 
A

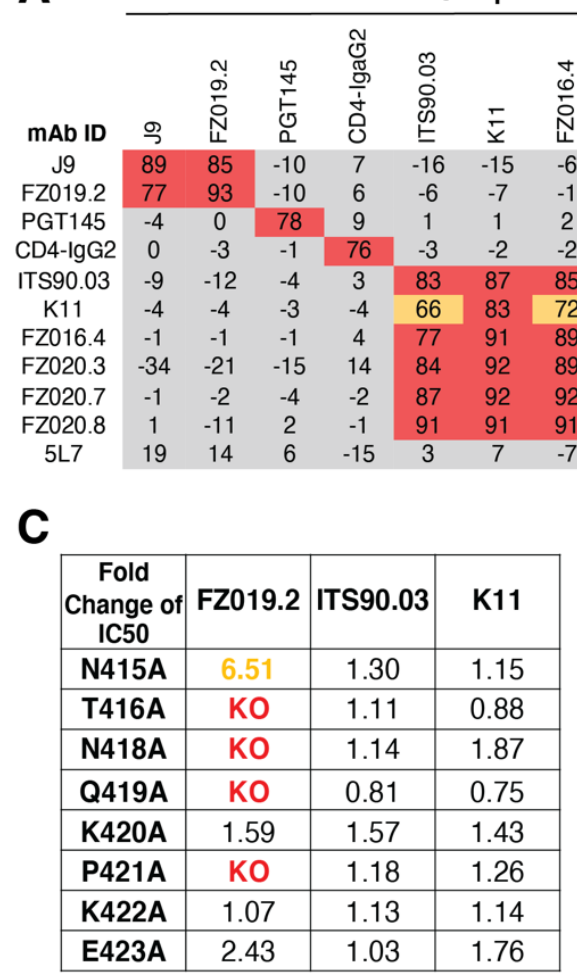

B


Maximum

Competition

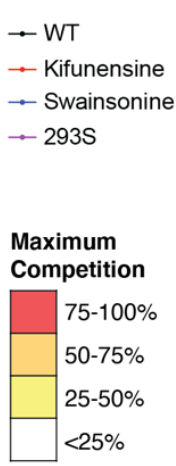

$\rightarrow W T$

- Swainsonine

$<25 \%$

E
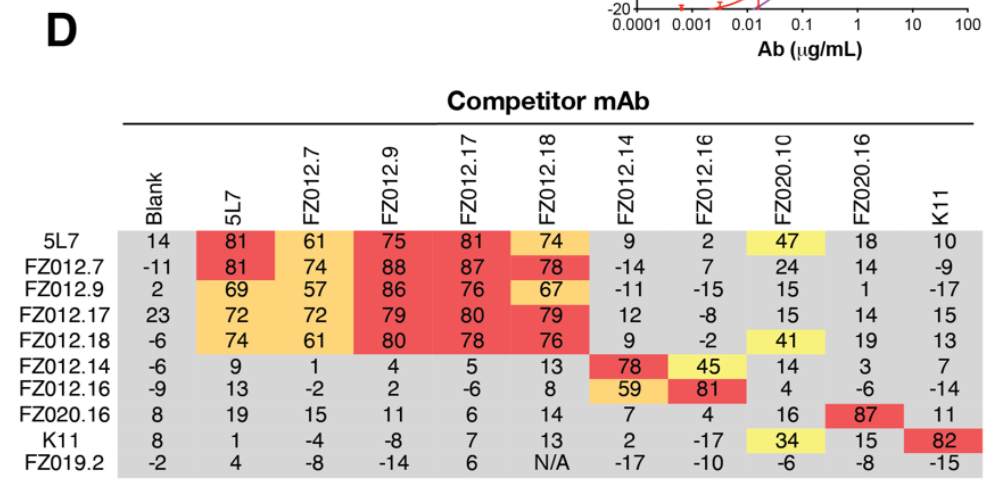

\begin{tabular}{|c|c|c|c|c|c|c|c|c|c|c|}
\hline mAb ID & Animal ID & $\mathbf{v}_{\mathrm{H}}$ & $D_{H}$ & $\mathbf{J}_{\mathrm{H}}$ & $\begin{array}{c}\mathrm{V}_{\mathrm{H}} \mathrm{SHM} \\
\mathrm{nt}(\%)\end{array}$ & CDRH3 & $\mathrm{v}_{\mathrm{L}}$ & $J_{\mathrm{L}}$ & $\begin{array}{c}\mathrm{V}_{\mathrm{H}} \mathrm{SHM} \\
\mathrm{nt}(\%)\end{array}$ & CDRL3 \\
\hline FZ012.7 & R11039 & IGHV4-AFB*01 & IGHD2-13*01 & IGHJ2-I1 & 5.74 & ARVTLSSWYFDL & LJI.Rh_IGLV3.32 & IGLJ1-S1 & 4.86 & QWWDGNTDHYI \\
\hline FZ012.9 & R11039 & IGHV4-AFB*01 & IGHD2-2*01 & IGHJ4*01 & 6.71 & ARVVLAAPYFDS & IGLV3-AED*06 & IGLJ2-S2 & 8.48 & QVWDGDSDQYI \\
\hline FZ012.14 & R11039 & IGHV1-AAU*01_S5608 & IGHD2-35*01 & LJI.Rh-IGHJ5.4 & 7.53 & ATLGGSCTGGVCRDYFEF & IGLV7-AAK*01 & IGLJ2-S2 & 12.37 & LWYYSGSGL \\
\hline FZ012.16 & R11008 & IGHV3-ADL*01 & IGHD2-35*01 & IGHJ4*01 & 10.74 & ARAGGCIAGVCSYFDS & LJI.Rh_IGKV2.71.a & IGKJ5-15 & 5.44 & GQGTHWPWT \\
\hline FZ012.17 & R11008 & LJI.Rh_IGHV3.141 & IGHD1-1*02 & LJI.Rh-IGHJ5.4 & 13.54 & TSLSLAGMDDSAEYFQF & IGLV1-ACN*02 & IGLJ3*01 & 4.86 & QSYDRTLDIHI \\
\hline FZ012.18 & R11008 & LJI.Rh_IGHV3.141 & IGHD2-2*01 & $\mathrm{IGHJ} 3^{*} 01$ & 13.54 & TTLSLAGMDDDAEYFVF & IGLV1-S30 & IGLJ3*01 & 4.15 & QSYDNGLNIHL \\
\hline FZ020.16 & R11008 & IGHV4-ABB*01_S8200 & IGHD2-13 & IGHJ5-1*01 & 12.67 & AKEYCKIPSCSSIPV & IGLV3-AEB*02 & IGLJ2-S2 & 6.60 & YSTDSSGNHGL \\
\hline
\end{tabular}

\section{F}

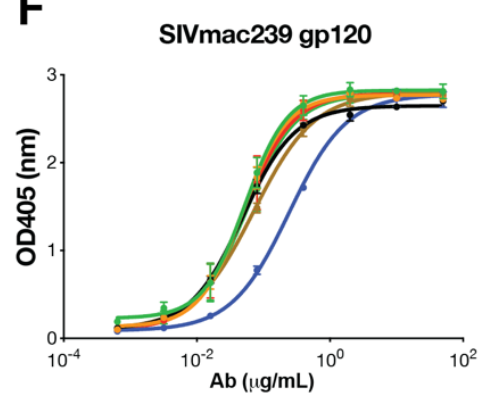

SIVmac239 gp120 dV1V2

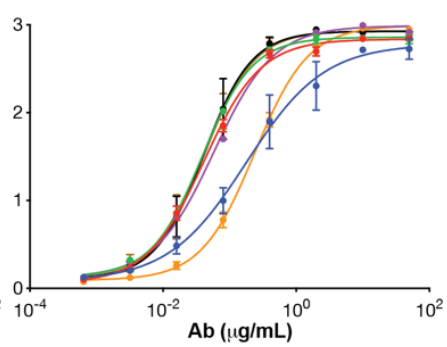



.

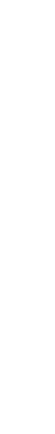



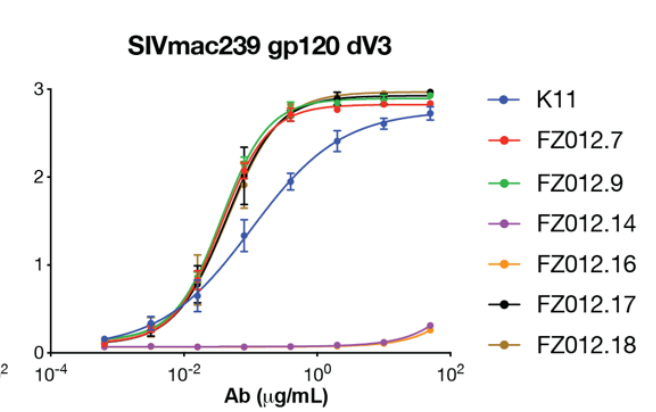

Figure S2. Characterization of SIVmac239 nAb and non-nAb epitope specificities. (A) ELISA competition between SIVmac239 nAbs, a SIVmac239 non-nAb 5L7, an HIV-1 bnAb PGT145, and CD4-IgG2 for binding to SIVmac239 SOSIP.664. Maximum percentage of competition was colored according to the key. (B) Representative neutralization curves of FZ019.2 and K11 against wildtype SIVmac239 virus, 293S-generated virus, and glycosidase inhibitor-treated viruses at the following concentrations: $25 \mu \mathrm{M}$ kifunensine and $20 \mu \mathrm{M}$ swainsonine, respectively. Each sample is tested in duplicate and error bars represent standard deviations. (C) Fold reduction of neutralization potency against alanine-scan variants at the V4 loop relative to wildtype SIVmac239 pseudovirus. KO: knockout of neutralization i.e. no neutralization measured at $50 \mu \mathrm{g} / \mathrm{mL}$ of $\mathrm{mAbs}$. (D) ELISA competition between SIVmac239 non-nAbs, 5L7, as well as nAbs K11 and FZ019.2 for binding to SIVmac239 SOSIP.664. Maximum percentage of competition was colored according to the key. (E) Immunogenetics of SIVmac239 non-nAb mAbs. Rhesus mAbs were annotated with a new rhesus germline database (Cottrell et al., 2020). (F) Representative ELISA binding curves of non-nAbs binding to SIVmac239 gp120, gp120 dV1V2, and gp120 dV1V2V3. Antibodies were colored according to the key. Each sample is tested in duplicate and error bars represent standard deviations. 
A

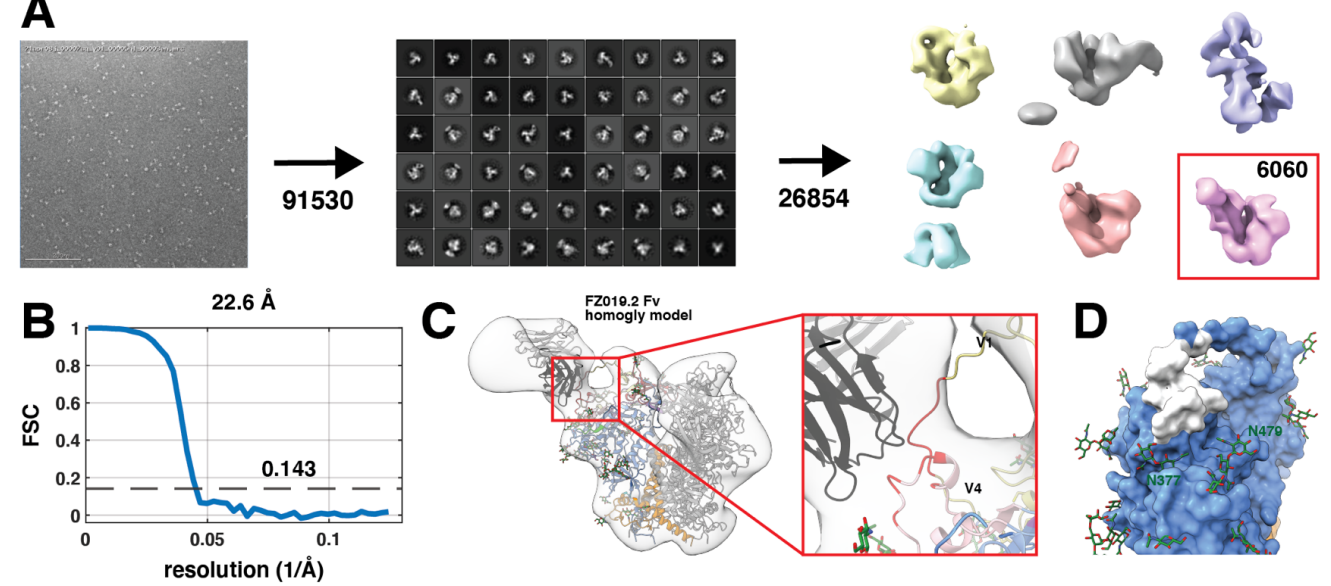

$\mathbf{E}$

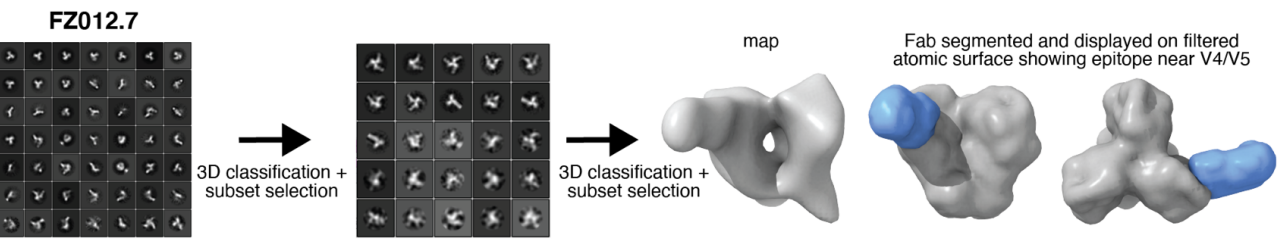

$\mathbf{F}$
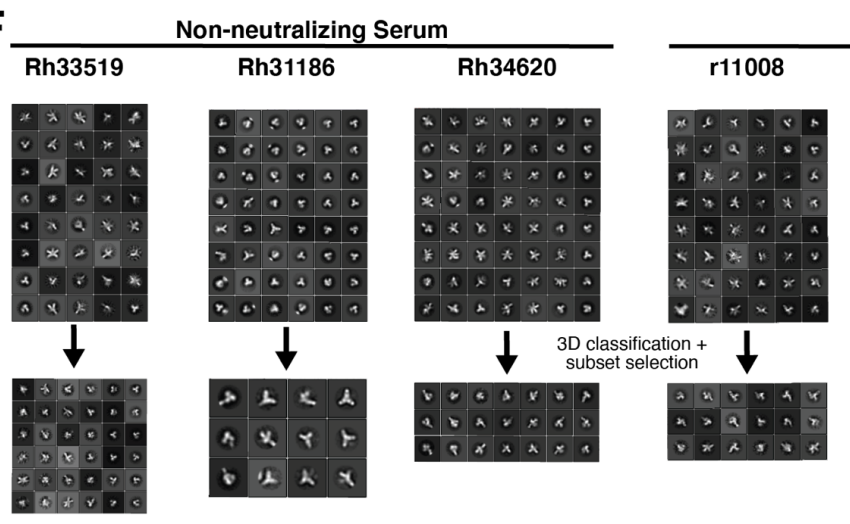

Neutralizing Serum
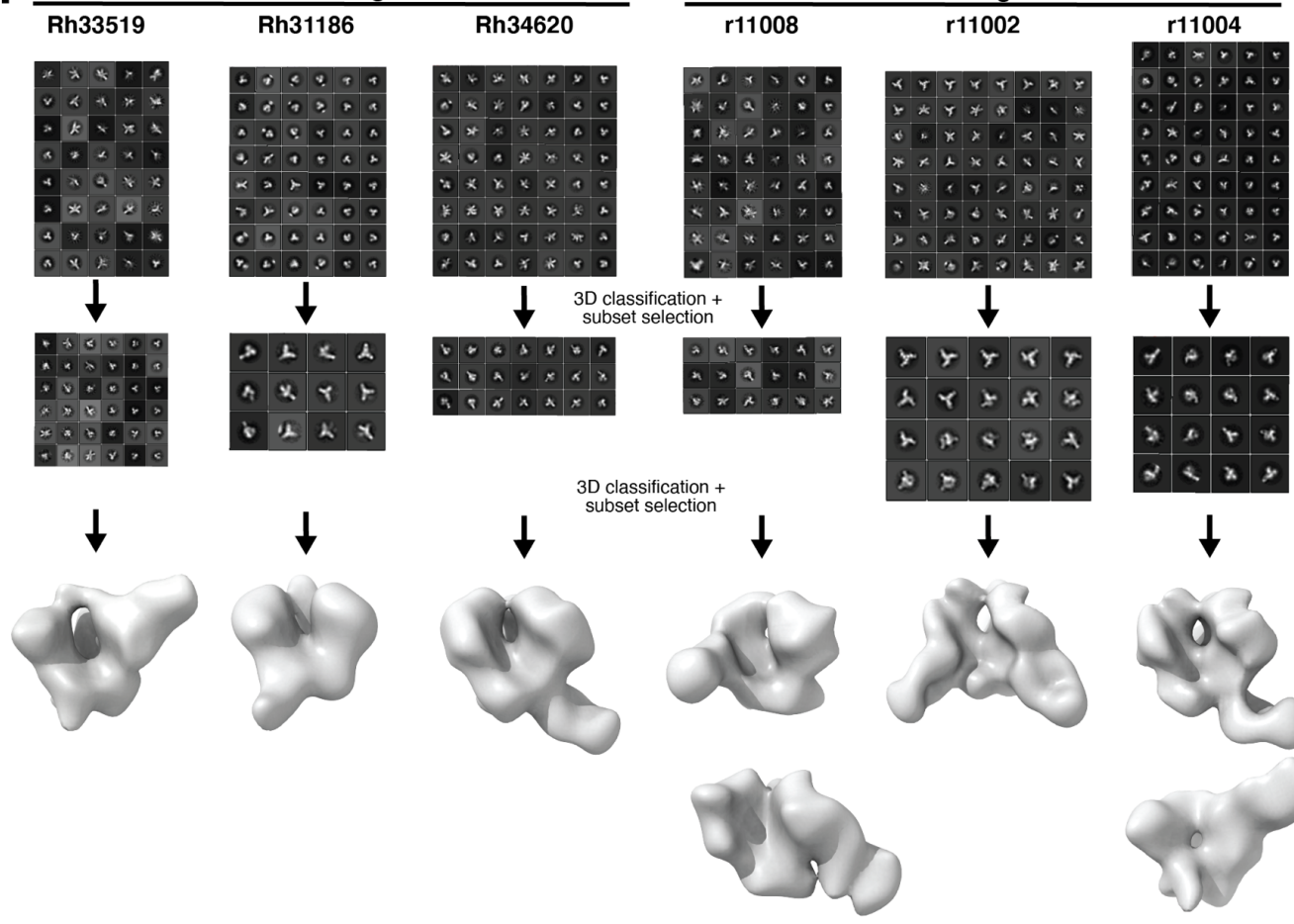

Figure S3. NSEM data processing workflows all mAb and polyclonal data. (A) Data processing workflow for FZ019.2 Fab bound to SIVmac239 SOSIP showing a representative raw micrograph along with 2-D and 3-D class averages and particle numbers indicated at each step. (B) Fourier Shell Correlation plot for the final 3-D reconstruction. (C) FZ019.2 epitope mapping using the cryo-EM structure and homology model of the Fab showing potential epitope residues located in the V1 and V4 loops colored in red. (D) Molecular surface representation of SIVmac239 gp120 showing FZ019.2 epitope in white. (E) Data processing workflow for FZ012.7 bound to the SIVmac239 trimer along with a segmentation of the Fab displayed on a molecular surface representation of the SIVmac239 trimer structure. (F) Data processing workflow for all polyclonal samples tested. 

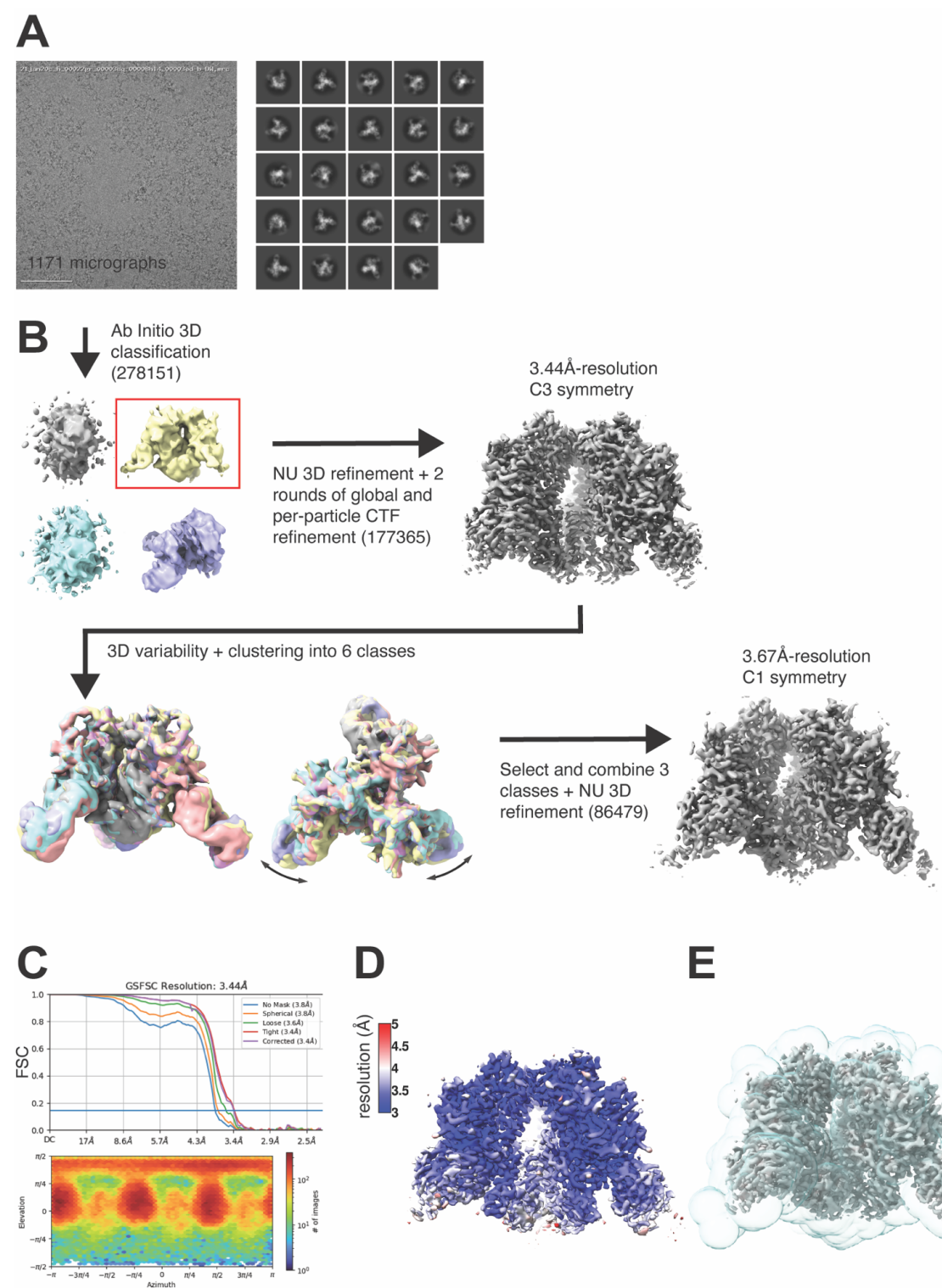

E $\quad \mathbf{F}=\mathrm{gp} 120 \square \mathrm{gp} 41 \amalg \mathrm{K} 11 \mathrm{lgG}$
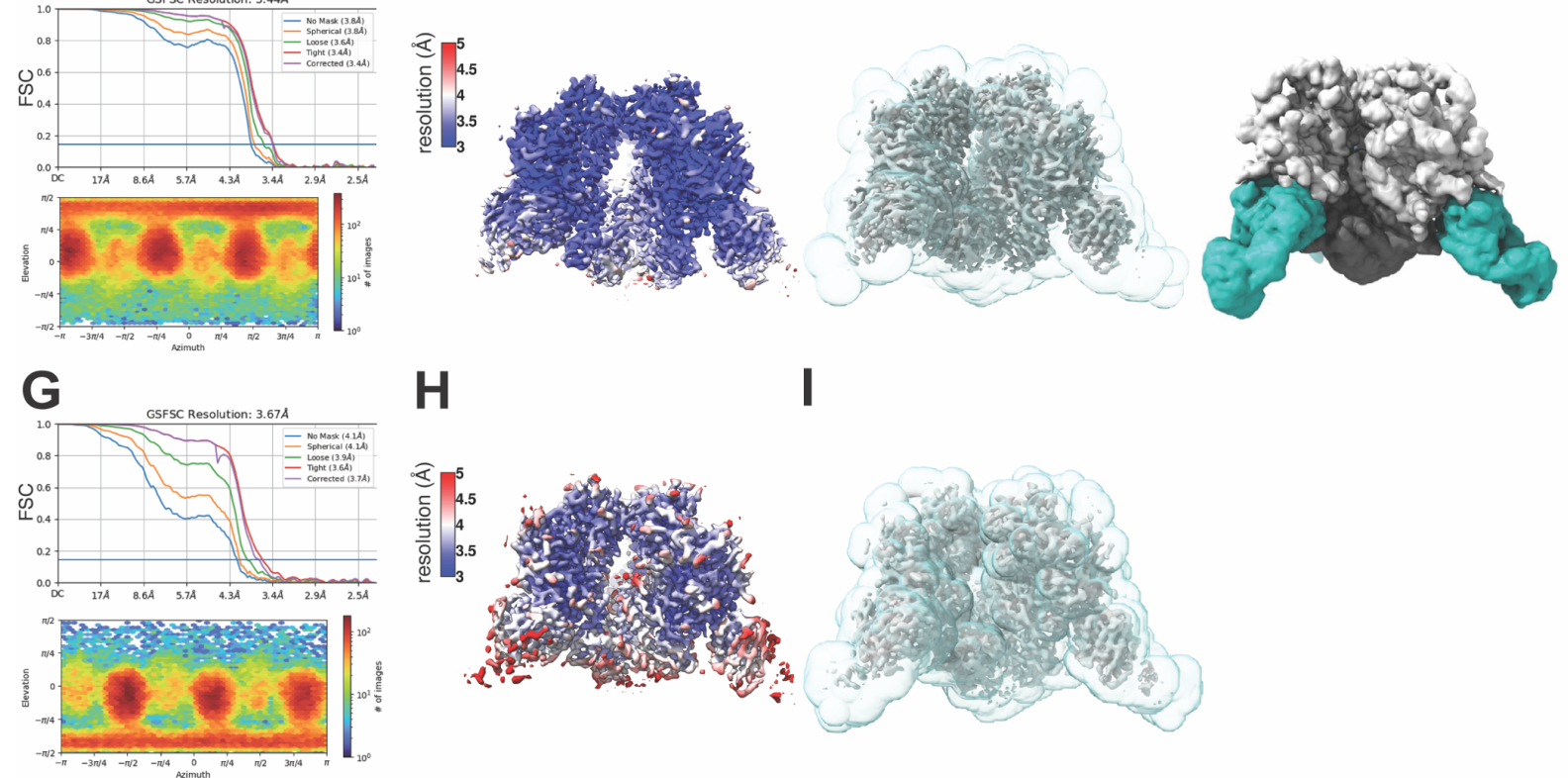

Figure S4. Cryo-EM data processing workflow. (A) Raw micrograph and representative 2-D class averages for the SIVmac239.K180S + K11 IgG cryo-EM dataset. (B) 3-D data processing workflow showing sorting by ab initio classification and 3-D variability analysis resulting in a C3 consensus refinement and asymmetric refinement from 3D variability clusters. (C) Fourier Shell Correlation and angular distribution plots for the C3 consensus refinement. (D) Local resolution analysis, (E) mask used for refinement, and (F) final segmented map. (G-I) Same as C-D but for asymmetric refinement. 

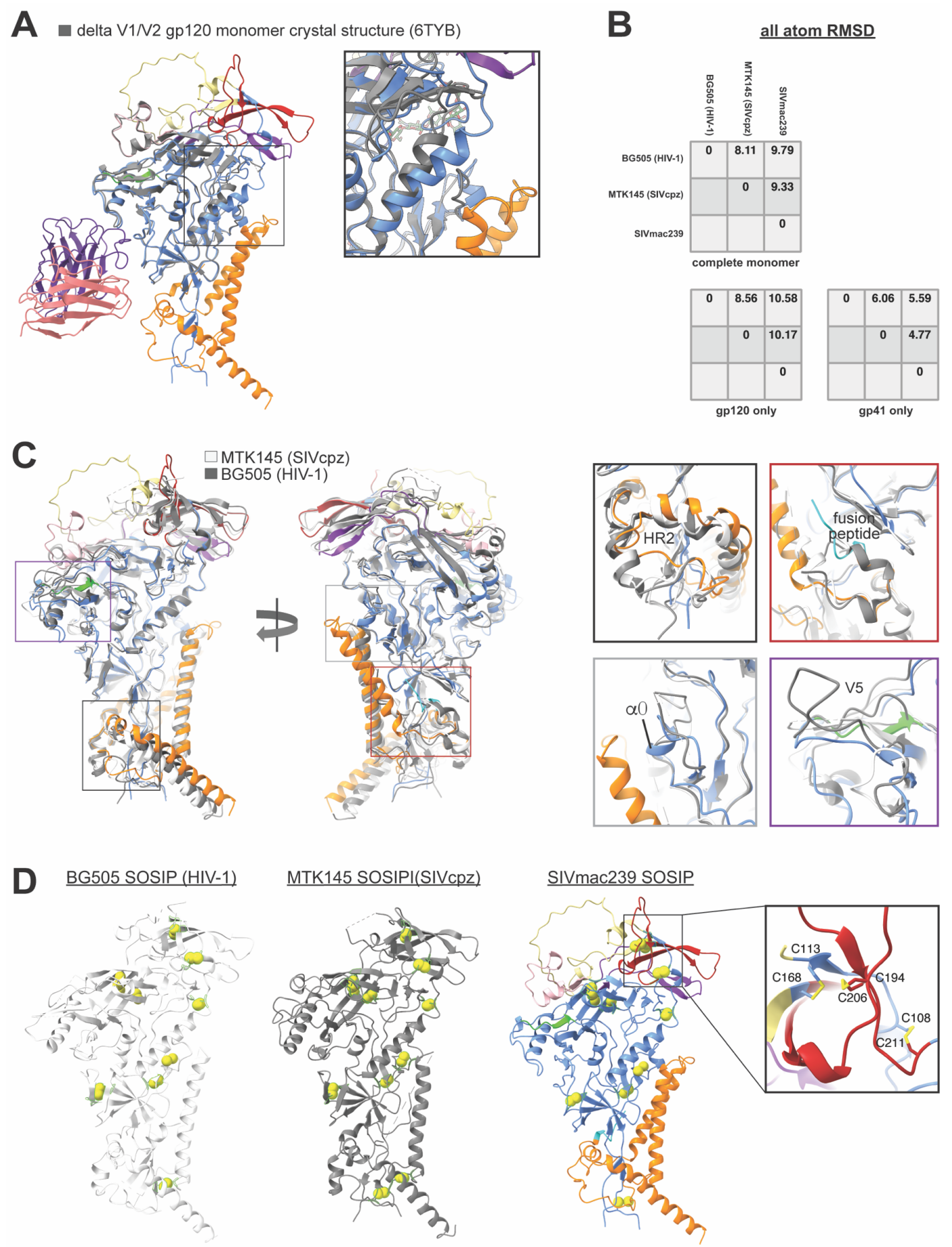

Figure S5. Extended structural analysis. (A) Alignment of our cryo-EM structure with the delta V1/N2 gp120 monomer crystal structure (PDB: 6TYB) showing close agreement outside of the trimerization and gp120-gp41 interfaces (close-up view). (B) All atom root mean squared deviations (RMSD) between all three structures. (C) Two views of the SIVmac239 monomer with MT145K SIVcpz and BG505 SOSIP.664 HIV-1 Env structures overlayed and aligned to gp120. Coloring the same as Figure 4. Close up views from designated regions in panel A with higher levels of structural variability between the three models. Specifically, the HR2 helical turn, fusion peptide, alpha 0, and V5 loop. (D) Detected (highlighted in green) and probable (not highlighted) disulfide bonds for all three models. Close up of the base of V1 and V2 loops showing the additional disulfide unique to SIVmac239. 




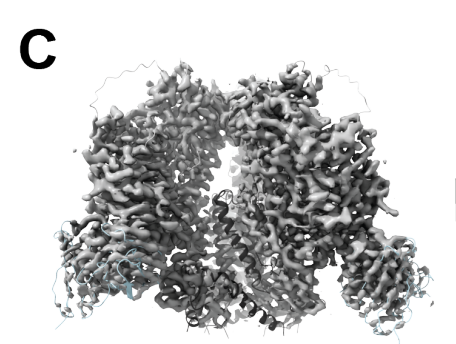

1. Build complete atomic model into cryo-EM map wihtout glycans

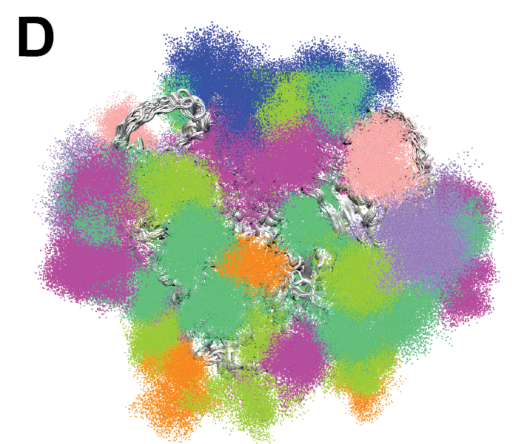



2. Generate a diverse set of protein models with Rosetta multicycle refinement and select the top ten scoring models to serve as scaffolds (removing Fab chains if present)

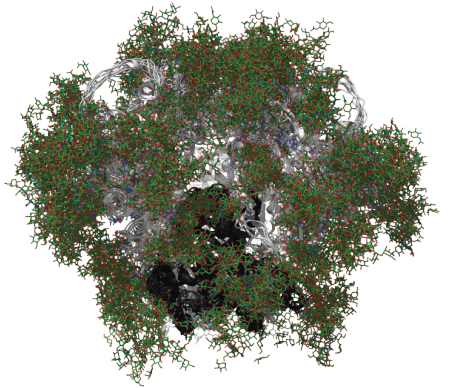

3. Build and relax 100 fully glycosylated models for each protein scaffold with ALLOSMOD using the most common glycoform at each site as determined by MS (1000 models total).

Figure S6. Extended glycan shield analysis. (A) Complete site-specific MS analysis showing the distribution of specific glycoforms for each PNGS along with gross percentages of each type of glycan (B). (C) Schematic overview of the modified HTAM pipeline using the cryo-EM map to guide the generation of protein scaffolds for subsequent glycan modeling. (D) All fully glycosylated models aligned to one another with glycans colored according to their type designated in the legend. 
A

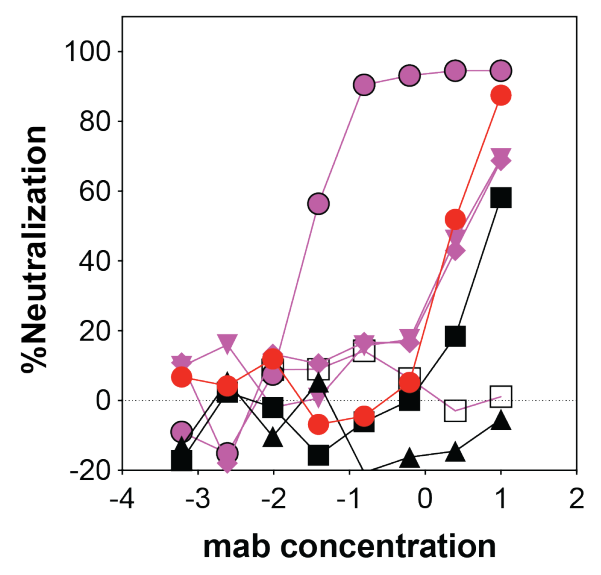

C

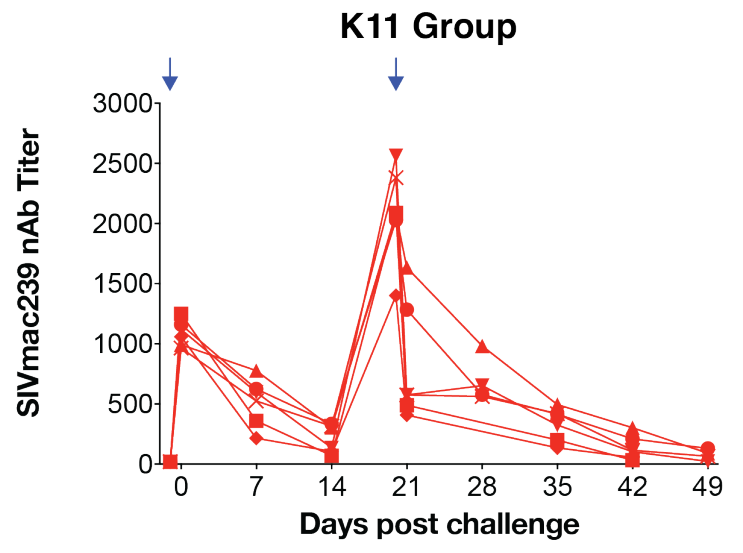

B

- $\mathrm{K} 11$

FZ019.2

$\therefore$ J9

- FZ020.1

- FZ020.3

- CD4 IgG2

$\square$ Den3
- r17034

$-r 17041$

- r17075

$\rightarrow \mathrm{r} 18014$

$\rightarrow \mathrm{r} 18022$

$\times$ rh2961

Figure S7. Macaque challenge study details. (A) Neutralization curves of K11 and FZ019.2 against SIVmac239 challenge virus. CD4-IgG2 was the positive control and Den3 was negative control. Antibodies are colored according to the key. (B) Plasma antibody concentrations in the K11 group (top) and 5L7-LS group (bottom). Rhesus plasma 5L7 was measured by ELISA binding to the C9 tag on the 5L7-LS antibody whereas K11 was measured by ELISA binding to SIVmac239 gp140 FT. Arrow represents antibody infusion. (C) Plasma SIVmac239 nAb titers in the K11 group. 


\begin{tabular}{cccccccc} 
& & \multicolumn{5}{c}{ SIVmac239 ELISA Binding EC } \\
mAb ID & Animal & (ug/mL) \\
\cline { 3 - 8 } & ID & SOSIP & gp140 FT & dV1V2V3 & gp120 & $\begin{array}{c}\text { gp120 } \\
\text { dV1V2 }\end{array}$ & $\begin{array}{c}\text { gp120 } \\
\text { dV3 }\end{array}$ \\
K11 & r11008 & 0.529 & 0.100 & 0.066 & 0.109 & 0.055 & 0.068 \\
FZ012.15 & r11008 & 0.611 & 0.077 & 0.037 & 0.058 & 0.036 & 0.062 \\
FZ020.1 & r11008 & 0.337 & 0.063 & 0.044 & 0.045 & 0.034 & 0.053 \\
FZ020.3 & r11008 & 0.226 & 0.031 & 0.042 & 0.034 & 0.036 & 0.053 \\
FZ020.5 & r11008 & 1.799 & 0.092 & 0.023 & 0.099 & 0.067 & 0.127 \\
FZ016.3 & r11002 & 1.130 & 0.050 & 0.028 & 0.070 & 0.088 & 0.245 \\
FZ016.4 & r11002 & 0.411 & 0.033 & 0.022 & 0.039 & 0.040 & 0.089 \\
FZ016.5 & r11002 & 0.669 & 0.047 & 0.027 & 0.048 & 0.070 & 0.176 \\
FZ020.7 & r11002 & 0.923 & 0.042 & 0.022 & 0.067 & 0.079 & 0.113 \\
FZ020.8 & r11002 & 0.958 & 0.040 & 0.021 & 0.064 & 0.064 & 0.109 \\
FZ019.2 & r11039 & 0.334 & 0.527 & $>50$ & 2.752 & $>50$ & 1.159 \\
J9 & r11039 & 0.084 & 1.158 & $>50$ & N/A & $>50$ & N/A \\
ITS90.03 & N/A & 0.289 & 0.047 & 0.043 & 0.028 & 0.022 & 0.040
\end{tabular}

Table S1. Mapping SIVmac239 mAb binding specificities by ELISA. 


\begin{tabular}{|c|c|c|}
\hline & $\begin{array}{c}\text { SIVmac239.K180S } \\
\text { SOSIP + FZ019.2 Fab } \\
\text { (NSEM) }\end{array}$ & $\begin{array}{c}\text { SIVmac239.K180S } \\
\text { SOSIP + K11 IgG } \\
\text { (Cryo-EM) }\end{array}$ \\
\hline & EMDB: EMD-25621) & EMDB: EMD-25676 \\
\hline & PDB ID: 7T2P & PDB ID: 7T4G \\
\hline \multicolumn{3}{|c|}{ Data Collection and Processing } \\
\hline Electron microscope & Titon Krios & Titon Krios \\
\hline Electron detector & K2 Summit & K2 Summit \\
\hline Magnification & 36,000 & 36,000 \\
\hline Voltage $(\mathrm{kV})$ & 200 & 200 \\
\hline Electron exposure $\left(\mathrm{e}-/ \AA^{2}\right)$ & 5 & 5 \\
\hline \multicolumn{3}{|l|}{ Defocus range $(\mu \mathrm{m})$} \\
\hline Pixel Size $(\AA)$ & 1.15 & 1.15 \\
\hline Symmetry imposed & $\mathrm{C} 3$ & C1 \\
\hline Initial particle images (no.) & 278,151 & 278,151 \\
\hline Final particle images (no.) & 177,365 & 86,479 \\
\hline Map resolution $(\AA)$ & 3.47 & 3.67 \\
\hline FSC threshold & 0.143 & 0.143 \\
\hline Map sharpening $B$ factor $\left(\AA^{2}\right)$ & 101.8 & 127.3 \\
\hline \multicolumn{3}{|l|}{ Model building and refinement } \\
\hline Initial models used & (6TYB,6X9V) & C1 Map \\
\hline \multicolumn{3}{|l|}{ Model composition } \\
\hline Protein Chains & 4 & 12 \\
\hline Protein Residues & 873 & 2619 \\
\hline Ligands & 56 & 176 \\
\hline \multicolumn{3}{|l|}{ R.m.s. deviations } \\
\hline Bond Lengths $(\AA)$ & 0.021 & 0.021 \\
\hline Bond angles $\left(^{\circ}\right)$ & 1.816 & 1.771 \\
\hline \multicolumn{3}{|l|}{ Ramachandran plot } \\
\hline Favored (\%) & 95.49 & 95.8 \\
\hline Disallowed (\%) & 1.39 & 1.35 \\
\hline \multicolumn{3}{|l|}{ Validation } \\
\hline MolProbity score & 1.09 & 1.12 \\
\hline Clashscore & 0.93 & 1.17 \\
\hline Poor rotamers (\%) & 0.39 & 0.26 \\
\hline EMRinger score & 2.06 & 1.87 \\
\hline Map-model cross correlation & 0.79 & 0.82 \\
\hline CaBLAM outliers (\%) & 0.12 & 3.66 \\
\hline
\end{tabular}

Table S2. Cryo-EM data and atomic model statistics. 
bioRxiv preprint doi: https://doi.org/10.1101/2021.12.22.473937; this version posted December 23, 2021. The copyright holder for this preprint

(which was not certified by peer review) is the author/funder. All rights reserved. No reuse allowed without permission.

\begin{tabular}{|c|c|c|c|c|c|}
\hline Epitope & Biotinylated Peptide ID & Sequence & Epitope & Biotinylated Peptide ID & Sequence \\
\hline V1V2 & SIVmac239_Env_Peptide1 & NKSETDRWGLTKSIT & gp41 & SIVmac239_Env_Peptide39 & GVFVLGFLGFLATAG \\
\hline V1V2 & SIVmac239_Env_Peptide2 & DRWGLTKSITTTAST & gp41 & SIVmac239_Env_Peptide40 & GFLGFLATAGSAMGA \\
\hline V1V2 & SIVmac239_Env_Peptide3 & TKSITTTASTTSTTA & gp41 & SIVmac239_Env_Peptide41 & LATAGSAMGAASLTL \\
\hline V1V2 & SIVmac239_Env_Peptide4 & TTASTTSTTASAKVD & gp41 & SIVmac239_Env_Peptide42 & SAMGAASLTLTAQSR \\
\hline V1V2 & SIVmac239_Env_Peptide5 & TSTTASAKVDMVNET & gp41 & SIVmac239_Env_Peptide43 & ASLTLTAQSRTLLAG \\
\hline V1V2 & SIVmac239_Env_Peptide6 & SAKVDMVNETSSCIA & gp41 & SIVmac239_Env_Peptide44 & TAQSRTLLAGIVQQQ \\
\hline V1V2 & SIVmac239_Env_Peptide7 & MVNETSSCIAQDNCT & gp41 & SIVmac239_Env_Peptide45 & TLLAGIVQQQQQLLD \\
\hline V1V2 & SIVmac239_Env_Peptide8 & SSCIAQDNCTGLEQE & gp41 & SIVmac239_Env_Peptide46 & IVQQQQQLLDVVKRQ \\
\hline V1V2 & SIVmac239_Env_Peptide9 & QDNCTGLEQEQMISC & gp41 & SIVmac239_Env_Peptide47 & QQLLDVVKRQQELLR \\
\hline V1V2 & SIVmac239_Env_Peptide10 & GLEQEQMISCKFNMT & gp41 & SIVmac239_Env_Peptide48 & WKRQQELLRLTWWG \\
\hline V1V2 & SIVmac239_Env_Peptide11 & QMISCKFNMTGLKRD & gp41 & SIVmac239_Env_Peptide49 & QELLRLTVWGTKNLQ \\
\hline V1V2 & SIVmac239_Env_Peptide12 & KFNMTGLKRDKKKEY & gp41 & SIVmac239_Env_Peptide50 & LTVWGTKNLQTRVTA \\
\hline V1V2 & SIVmac239_Env_Peptide13 & GLKRDKKKEYNETWY & gp41 & SIVmac239_Env_Peptide51 & TKNLQTRVTAIEKYL \\
\hline V1V2 & SIVmac239_Env_Peptide14 & KKKEYNETWYSADLV & gp41 & SIVmac239_Env_Peptide52 & TRVTAIEKYLKDQAQ \\
\hline V1V2 & SIVmac239_Env_Peptide15 & NETWYSADLVCEQGN & gp41 & SIVmac239_Env_Peptide53 & IEKYLKDQAQLNAWG \\
\hline V1V2 & SIVmac239_Env_Peptide16 & SADLVCEQGNNTGNE & gp41 & SIVmac239_Env_Peptide54 & KDQAQLNAWGCAFRQ \\
\hline V1V2 & SIVmac239_Env_Peptide17 & CEQGNNTGNESRCYM & gp41 & SIVmac239_Env_Peptide55 & LNAWGCAFRQVCHTT \\
\hline V1V2 & SIVmac239_Env_Peptide18 & NTGNESRCYMNHCNT & gp41 & SIVmac239_Env_Peptide56 & CAFRQVCHTTVPWPN \\
\hline V3 & SIVmac239_Env_Peptide19 & RRPGNKTVLPVTIMS & gp41 & SIVmac239_Env_Peptide57 & VCHTTVPWPNASLTP \\
\hline V3 & SIVmac239_Env_Peptide20 & KTVLPVTIMSGLVFH & gp41 & SIVmac239_Env_Peptide58 & VPWPNASLTPKWNNE \\
\hline V3 & SIVmac239_Env_Peptide21 & VTIMSGLVFHSQPIN & gp41 & SIVmac239_Env_Peptide59 & ASLTPKWNNETWQEW \\
\hline V3 & SIVmac239_Env_Peptide22 & GLVFHSQPINDRPKQ & gp41 & SIVmac239_Env_Peptide60 & KWNNETWQEWERKVD \\
\hline V3 & SIVmac239_Env_Peptide23 & SQPINDRPKQAWCWF & gp41 & SIVmac239_Env_Peptide61 & TWQEWERKVDFLEEN \\
\hline V4 & SIVmac239_Env_Peptide24 & KMNWFLNWVEDRNTA & gp41 & SIVmac239_Env_Peptide62 & ERKVDFLEENITALL \\
\hline V4 & SIVmac239_Env_Peptide25 & LNWVEDRNTANQKPK & gp41 & SIVmac239_Env_Peptide63 & FLEENITALLEEAQI \\
\hline V4 & SIVmac239_Env_Peptide26 & DRNTANQKPKEQHKR & gp41 & SIVmac239_Env_Peptide64 & ITALLEEAQIQQEKN \\
\hline V4 & SIVmac239_Env_Peptide27 & NQKPKEQHKRNYVPC & gp41 & SIVmac239_Env_Peptide65 & EEAQIQQEKNMYELQ \\
\hline V5 & SIVmac239_Env_Peptide28 & WIDGNQ & gp41 & SIVmac239_Env_Peptide66 & QQEKNMYELQKLNSW \\
\hline $\mathrm{C} 3$ & SIVmac239_Env_Peptide29 & CWFGGKWKDAIKEVK & gp41 & SIVmac239_Env_Peptide67 & MYELQKLNSWDVFGN \\
\hline $\mathrm{C} 3$ & SIVmac239_Env_Peptide30 & KWKDAIKEVKQTIVK & gp41 & SIVmac239_Env_Peptide68 & KLNSWDVFGNWFDLA \\
\hline C3 & SIVmac239_Env_Peptide31 & IKEVKQTIVKHPRYT & gp41 & SIVmac239_Env_Peptide69 & DVFGNWFDLASWI \\
\hline $\mathrm{C} 3$ & SIVmac239_Env_Peptide32 & QTIVKHPRYTGTNNT & & & \\
\hline $\mathrm{C} 3$ & SIVmac239_Env_Peptide33 & HPRYTGTNNTDKINL & & & \\
\hline $\mathrm{C} 3$ & SIVmac239_Env_Peptide34 & GTNNTDKINLTAPGG & & & \\
\hline $\mathrm{C} 3$ & SIVmac239_Env_Peptide35 & DKINLTAPGGGDPEV & & & \\
\hline $\mathrm{C} 3$ & SIVmac239_Env_Peptide36 & TAPGGGDPEVTFMWT & & & \\
\hline $\mathrm{C} 3$ & SIVmac239_Env_Peptide37 & GDPEVTFMWTNCRGE & & & \\
\hline C3 & SIVmac239_Env_Peptide38 & TFMWTNCRGEFLYCK & & & \\
\hline
\end{tabular}

Table S3. SIVmac239 Env 15-mer overlapping peptide sequences. 


\begin{tabular}{|c|c|c|c|c|}
\hline Group & ID & Sex & MHC & Age (years) \\
\hline Group 1 5L7-LS & rh2967 & female & - & 4.88 \\
\hline Group 1 5L7-LS & r17107 & male & $A^{*} 02+$ & 3.30 \\
\hline Group 1 5L7-LS & r18011 & female & $A^{*} 02+$ & 2.96 \\
\hline Group 1 5L7-LS & r18025 & male & $A^{*} 02-$ & 2.83 \\
\hline Group 1 5L7-LS & r18044 & male & $\mathrm{A}^{*} 02+$ & 2.72 \\
\hline Group 1 5L7-LS & rh2962 & female & - & 3.67 \\
\hline Group 2 K11 & r17034 & male & $\mathrm{A}^{*} 02-$ & 3.75 \\
\hline Group 2 K11 & r17041 & female & $A^{*} 02-$ & 3.71 \\
\hline Group 2 K11 & r17075 & female & $A^{*} 02-$ & 3.45 \\
\hline Group 2 K11 & r18014 & male & $A^{*} 02-$ & 2.93 \\
\hline Group 2 K11 & r18022 & male & $A^{*} 02+$ & 2.88 \\
\hline Group 2 K11 & rh2961 & female & - & 4.82 \\
\hline Group 3 & r17068 & male & $A^{*} 02-$ & 3.55 \\
\hline Group 3 & r17024 & male & - & 3.79 \\
\hline Group 3 & r17035 & male & $A^{\star} 02-$ & 3.75 \\
\hline Group 3 & r17060 & male & $A^{*} 02+$ & 3.59 \\
\hline Group 3 & r18017 & male & $A^{*} 02+$ & 2.92 \\
\hline Group 3 & rh2751 & female & - & 5.78 \\
\hline
\end{tabular}

Table S4. Genetic background of animals in the challenge study. 